A preliminary version of this paper appears in TCC 2016-A. This is the full version appearing as IACR ePrint Archive Report 2015/703.

\title{
Point-Function Obfuscation: A Framework and Generic Constructions
}

\author{
Minir Bellare ${ }^{\boxplus} \quad$ IgOrs StePanovi ${ }^{\square}$
}

March 2016

\begin{abstract}
We give a definitional framework for point-function obfuscation in which security is parameterized by a class of algorithms we call target generators. Existing and new notions are captured and explained as corresponding to different choices of this class. This leads to an elegant question: Is it possible to provide a generic construction, meaning one that takes an arbitrary class of target generators and returns a point-function obfuscator secure for it? We answer this in the affirmative with three generic constructions, the first based on indistinguishability obfuscation, the second on deterministic public-key encryption and the third on universal computational extractors. By exploiting known constructions of the primitives assumed, we obtain new pointfunction obfuscators, including many under standard assumptions. We end with a broader look that relates different known and possible notions of point function obfuscation to each other and to ours.
\end{abstract}

\footnotetext{
${ }^{1}$ Department of Computer Science \& Engineering, University of California San Diego, 9500 Gilman Drive, La Jolla, California 92093, USA. Email: mihir@eng.ucsd.edu. URL: http://cseweb.ucsd.edu/ ${ }^{\sim m i h i r / . ~ S u p p o r t e d ~ i n ~}$ part by NSF grants CNS-1116800, CNS-1228890 and CNS-1526801. This work was done in part while the author was visiting the Simons Institute for the Theory of Computing, supported by the Simons Foundation and by the DIMACS/Simons Collaboration in Cryptography through NSF grant CNS-1523467.

2 Department of Computer Science \& Engineering, University of California San Diego, 9500 Gilman Drive, La Jolla, California 92093, USA. Email: istepano@eng.ucsd.edu. URL: http://cseweb.ucsd.edu/ ${ }^{\text {istepano/. }}$ Supported in part by NSF grants CNS-1116800 and CNS-1228890.
} 


\section{Contents}

1 Introduction 2

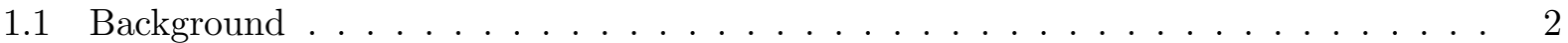

1.2 Contributions in brief $\ldots \ldots \ldots \ldots$

1.3 Definitional framework . . . . . . . . . . . . . . . . . . . .

1.4 Generic constructions . . . . . . . . . . . . . . . . . . . 目

1.5 Alternative notions and relations between notions . . . . . . . . . . . . . G

1.6 Discussion and further related work . . . . . . . . . . . . G

2 Notation and standard definitions $\quad$ 7

3 Point-function obfuscation framework 9

4 (d)iO for multi-circuit samplers

5 Generic constructions of PO

$5.1 \mathrm{PO}$ from $\mathrm{iO} \ldots \ldots \ldots \ldots \ldots \ldots \ldots \ldots$

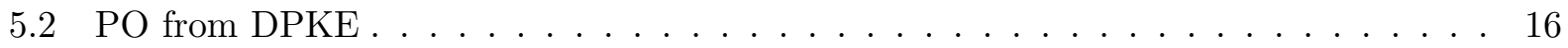

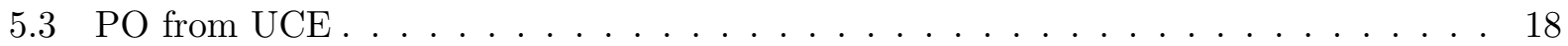

6 Alternative security notions for PO 20

A Computational correctness and PO from any UCE 


\section{Introduction}

In the theory of point-function obfuscation $(\mathrm{PO})$, there are many different goals and definitions. It is (at least to us) hard territory to navigate. Meanwhile, there are few constructions, and the ones that exist use strong assumptions. We try to bring some structure and unity to this area via a parameterized definitional framework, generic constructions and relations between definitions.

\subsection{Background}

A point function with target $k \in\{0,1\}^{*}$ is the circuit $\mathbf{I}_{k}$ that on input $k^{\prime} \in\{0,1\}^{|k|}$ returns 1 if $k^{\prime}=k$ and 0 otherwise. A point-function obfuscator Obf takes input $\mathbf{I}_{k}$ and returns another circuit $\overline{\mathrm{P}}$ that is functionally equivalent to $\mathbf{I}_{k}$, meaning on input $k^{\prime} \in\{0,1\}^{|k|}$ it also returns 1 if $k^{\prime}=k$ and 0 otherwise. Security requires that $\overline{\mathrm{P}}$ hides $k$. We now discuss prior security definitions and constructions.

DEFINITIONS. Security definitions of point-function obfuscation show consideration of many different issues and use different styles. Both definitions that allow auxiliary information [2.3, 35, 22.9, 14, 42, 21, 201] and ones that don't [2:3, 27, 41, 51, [25, [26, 6] have been considered. Sometimes security pertains only to a single target point [2.3, 27, 51, 35, 2.9, 20], other times it consider many simultaneous target points [41, 25, 11, 26, 14, 6, 42, [22]. Some definitions are simulation-based, splitting up into average-case [23, 27, 22, 26, 6] and worst-case [41, 51, 35, 25, 11, 14, 42]. (Simulation-based definitions can be further customized in a number of ways [50], some of which we discuss in Section [6.) Some definitions are indistinguishability based, with different formalizations in [2:3] and in [14, 42, 20, 21, 22]. Indistinguishability-based definitions require some kind of unpredictability of the target point, and there are variants here, for example whether the condition should be for polynomial-time or unbounded adversaries, and with negligible or sub-exponential advantage. And this list is not complete.

While many issues have been considered, the consideration has not been systematic, in the following sense. Think of issues (for example whether or not auxiliary information is present, one target or many, polynomial-time or unbounded predictability adversaries in the context of an indistinguishability-based definition, ...) as dimensions or axes in a multi-dimensional space. Then definitions in the literature can be seen as capturing some points in this space. But there is no systematic attempt to look in some unified way at all the points in this space. This is what we will attempt to do.

Constructions. Canetti [2:3] constructs a point-function obfuscator for a single target point, achieving an indistinguishability notion he defines that we call SIND. (In Section 6 we give a parameterized version of SIND.) Canetti [2:3] proves the security of his construction for three different security levels, each using a variant of the DDH assumption. The security with respect to auxiliarity information is proven under the assumption that is called DHI III in [23]. It was reformulated in [14], and that version was called AI-DHI (Auxiliary-Input Diffie-Hellman Inversion) by [II0], who showed that it cannot co-exist with an assumption that VGBO (Virtual Grey Box Obfuscation) [11, ए2] exists for all circuits. That doesn't mean it fails (perhaps VGBO does not exist) but, conservatively, it might be nice to be able to base PO on assumptions not in contention with other assumptions or conjectures. Wee [5] provides a PO construction based on a fixed permutation about which a novel, strong uninvertibility assumption is made. He only proves security in the absence of auxiliary information, and GK [35] show that the construction does not in fact provide security in the presence of auxiliary information. However BP [14] specify an extension of Wee's construction with a family of permutations rather than a fixed one, and show, under a novel 
assumption called Assumption 2.1 in their paper, that it achieves security with targets that are hard to predict given the auxiliary information. BP [14] explain that Assumption 2.1 asks for (a weak form of) extractability, making it a strong assumption in light of the impossibility of related extractable primitives [1:3]. DKL [2.9] use a novel assumption they call LSN to give a construction for targets that are exponentially hard to predict given the auxiliary information. BHK [6] give a construction for statistically hard to predict targets and no auxiliary information based on a multi-key version of their UCE assumption. There are simple constructions in the ROM [4I].

In summary, there are few constructions and those that exist use strong and sometimes novel assumptions. Also, each construction achieves a different variant of the goal, and it is hard to visualize, or say in a concise way, what has been done. The framework that we now discuss provides language to do this.

\subsection{Contributions in brief}

We pick one, simple indistinguishability-based definitional template. Using this, we provide a framework parameterized by a class $\mathbf{X}$ of objects we call target generators, giving a definition of what it means for a point-function obfuscator to be $\operatorname{IND}[\mathbf{X}]$ secure. This allows us to recover and explain different notions in the literature as each corresponding to a choice of $\mathbf{X}$, and also obtain many natural new ones, points in the above-mentioned multi-dimensional space that had not been explicitly considered.

This taxonomy leads to a compelling and general new question: Is it possible to find a generic construction, meaning a compiler that given an arbitrary $\mathbf{X}$ returns a point-function obfuscator secure relative to it? We answer this in the affirmative by providing three such generic constructions. As a consequence we obtain new constructions for both old and new forms of PO.

We then step back to consider other definitions of PO. These include existing simulation and indistinguishability style notions, as well as a new, semantic security style definition emanating from a connection to DPKE (Deterministic Public-Key Encryption) [3]. We formulate these also in a parameterized framework and then provide relations (implications and separations) between these notions and our IND notion.

We now look at these three contributions in more detail.

\subsection{Definitional framework}

Recall that a point-function obfuscator Obf takes input $\mathbf{I}_{k}$ and returns another circuit $\overline{\mathrm{P}}$ that is functionally equivalent to $\mathbf{I}_{k}$. Security requires that $\overline{\mathrm{P}}$ hides $k$. We define a target generator $\mathbf{X}$ as a polynomial-time algorithm that on input the security parameter returns a vector $\mathbf{k}$ of target points together with auxiliary information $a$. We measure security of a candidate point-function obfuscator Obf relative to $\mathrm{X}$. To do this, we associate to an adversary $\mathcal{A}$ its advantage $\operatorname{Adv}_{\mathrm{Obf}, \mathrm{X}, \mathcal{A}}^{\text {ind }}(\cdot)$ in guessing the challenge bit $b$ in the following game. We run $\mathbf{X}$ to get $(\mathbf{k}, a)$. We let $\overline{\mathbf{P}}$ be the vector obtained by independently obfuscating $\mathbf{I}_{k}$ for each of the targets $k$ from $\mathbf{k}(b=1)$ or by obfuscating the same number of random, independent targets $(b=0)$. The input to $\mathcal{A}$ is $\overline{\mathbf{P}}$ and $a$. Now we let $\mathbf{X}$ be a class (set) of target generators $\mathbf{X}$ and say that obfuscator Obf is IND $[\mathbf{X}]$-secure if $\operatorname{Adv}_{\mathrm{Obf}, \mathrm{X}, \mathcal{A}}^{\text {ind }}(\cdot)$ is negligible for all polynomial time $\mathcal{A}$ and all $\mathbf{X} \in \mathbf{X}$. Our definition of IND security is an extended, parameterized version of security definitions used for point-function obfuscation in [14, 42, 201, 21, [22]. See Section 3 for a formal definition.

What we have here is a notion of point-function obfuscation parameterized by a class of target generators. We view the latter as knobs. By turning these knobs (defining specific classes) we can 
capture specific restrictions, and by intersecting classes we can combine them, allowing us to speak precisely yet concisely about different variant notions that are unified in this way.

$\operatorname{IND}[\mathbf{X}]$-security is not achievable for all $\mathbf{X}$. For example, $\mathbf{X}$ could pick $\mathbf{k}[1]$ to be the string of all zeroes, and the adversary could test whether or not $\overline{\mathrm{P}}$ returns 1 on input that string. The minimal requirement for security is that the target points produced by $\mathbf{X}$ are unpredictable given $a$. In Section 3 we formalize a prediction game and advantage so that we can define the classes $\mathbf{X}^{\text {cup }}, \mathbf{X}^{\text {seup }}$ and $\mathbf{X}^{\text {sup }}$ of computationally, sub-exponentially and statistically unpredictable target generators. We let $\mathbf{X}^{q(\cdot)}$ denote the class of target generators outputting $q(\cdot)$ target points and $\mathbf{X}^{\varepsilon}$ the class of target generators that produce no auxiliary information. (Formally it is the empty string.)

The framework allows us to capture prior goals in compact way. $\operatorname{IND}\left[\mathbf{X}^{\text {cup }} \cap \mathbf{X}^{\varepsilon} \cap \mathbf{X}^{1}\right]$ is plain point-function obfuscation [2:3, 27, 41, 51] , where there is just one target point, no auxiliary information, and unpredictability is computational. $\operatorname{IND}\left[\mathbf{X}^{\text {cup }} \cap \mathbf{X}^{1}\right]$ is $\operatorname{AIPO}[23$, 35, 14, 20], where there is again one target point, but auxiliary information is now present, while unpredictability continues to be computational. IND $\left[\mathbf{X}^{\text {cup }}\right]$ is composable AIPO [25, 14], where there are many arbitrarily correlated target points, auxiliary information is present, and unpredictability is computational. DKL [2.9] achieve IND $\left[\mathbf{X}^{\text {sup }} \cap \mathbf{X}^{1}\right]$, where there is a single target that is statistically hard to predict given the auxiliary information. BHK [6] achieve $\operatorname{IND}\left[\mathbf{X}^{\sup } \cap \mathbf{X}^{\varepsilon}\right]$, where there are multiple targets, unpredictability is statistical, and there is no auxiliary information. Other prior notions can be captured in similar ways, and many natural new notions emerge as well.

\subsection{Generic constructions}

As we saw above, constructions so far have been ad hoc, targeting different security goals and using strong, novel assumptions to achieve them. The above framework allows us to frame a compelling question, namely whether there are generic constructions. By this we mean that we are handed an arbitrary class $\mathbf{X}$ of target generators and asked to craft an obfuscator that is $\mathrm{IND}[\mathbf{X}]$-secure. If we can do this, we can, in one step, obtain constructions for a wide variety of forms of PO, not only ones considered in the past, but also new ones.

We provide three such generic constructions. The first is based on indistinguishability obfuscation, the second on deterministic public-key encryption and the third on (multi-key) UCE.

One natural objection at this point is that we know that $\operatorname{IND}[\mathbf{X}]$ is not achievable for some choices of $\mathbf{X}$. For example, assuming iO, this is true for $\mathbf{X}=\mathbf{X}^{\text {cup }}$, meaning composable PO. (This follows by combining [20, 24].) So how can our constructions achieve $\operatorname{IND}[\mathbf{X}]$ for any given $\mathbf{X}$ ? In fact, they do, and this, interestingly, yields new negative results, ruling out the primitives we start from for those particular values of $\mathbf{X}$. We will explain further below.

PO FROM IO. The emergence of candidate constructions for iO (indistinguishability obfuscation) [33, 46, 12, 34] raised a natural hope, namely that one could obtain PO from iO. But this has not happened. Despite the many powerful applications of $\mathrm{iO}$, constructing point-function obfuscation from it has surprisingly evaded effort.

We show that $\mathrm{iO}$ plus a OWF yields PO. More precisely, for any class of target generators $\mathbf{X}$ we show $\mathrm{iO}+\mathrm{OWF}[\mathbf{X}] \Rightarrow \operatorname{IND}[\mathbf{X}]$ : Given iO and a family of functions that is one-way relative to $\mathbf{X}$ as defined in Section 5. . we can construct an obfuscator that is $\operatorname{IND}[\mathbf{X}]$-secure. The construction, result and proof are in Section $5 . \mathbf{D}$. The idea is that to obfuscate $\mathbf{I}_{k}$ we pick at random a key $f k$ for the OWF $\mathrm{F}$ (formally, the latter is a family of functions) and let $y=\mathrm{F}(f k, k)$. We consider the circuit $\mathrm{C}$ that hardwires $f k, y$ and on input $k^{\prime}$ returns 1 if $\mathrm{F}\left(f k, k^{\prime}\right)=y$ and 0 otherwise. We then apply an indistinguishability obfuscator to $\mathrm{C}$ to produce the obfuscated point function. The security proof is a sequence of hybrids. Although we assume only iO, we exploit diO [2, 16, T] in 
the proof in a manner similar to $[9]$. We will need it for circuits that differ only on one input, and in this case the result of $\mathrm{BCP}[16]$ says that an iO-secure obfuscator is also diO-secure, so the assumption remains $\mathrm{iO}$. As part of the proof we state and prove a lemma reducing (d)iO on polynomially-many, related circuits to the usual single-circuit case. We note that to guarantee the usual (perfect) correctness condition of a PO, we require the OWF to be injective.

We highlight the simplest case of this result as still being novel and of interest. Namely, given iO and an ordinary injective OWF, we achieve plain point-function obfuscation, $\operatorname{IND}\left[\mathbf{X}^{\operatorname{cup}} \cap \mathbf{X}^{\varepsilon} \cap \mathbf{X}^{1}\right]$ in our notation. Previous constructions have been under assumptions that at this point seem less accepted than $\mathrm{iO}$, and Wee [51] gives various arguments as to why this goal is hard under standard assumptions. Also on the negative side, combining our result with [20, [24] allows us, under iO, to rule out $\mathrm{OWF}\left[\mathbf{X}^{\text {cup }}\right]$ (one-way functions secure for polynomially-many correlated inputs that are computationally unpredictable in the presence of auxiliary information), at least in the injective case.

PO FROM DPKE. Deterministic public key encryption (DPKE) [3] was motivated by applications to efficient searchable encryption [3]. It cannot provide IND-CPA security. Instead, BBO [3] provide a definition of a goal called PRIV which captures the best-possible security that encryption can provide subject to being deterministic. At this point many constructions of DPKE are known for various variant goals [3, 15, 4, 17, 32, 5, 52, 54, 48, 44, 40].

We show how to leverage these for point-function obfuscation via our second generic construction. We show that PRIV1 $[\mathbf{X}] \Rightarrow \operatorname{IND}[\mathbf{X}]$. That is, given a deterministic public-key encryption scheme that is PRIV1 secure relative to $\mathbf{X}$ we can build a point-function obfuscator secure relative to the same class in a simple and natural way. Namely to obfuscate $\mathbf{I}_{k}$ we pick at random a public key $p k$ and the associated secret key sk for the DPKE scheme and let $c$ be the encryption of $k$ under $p k$. The point-function obfuscation is the circuit $\mathrm{C}$ that hardwires $p k, c$ and on input $k^{\prime}$, returns 1 if the encryption of $k^{\prime}$ under pk equals $c$, and 0 otherwise. The fact that the encryption is deterministic is used crucially to define the circuit. (The latter must be deterministic.) The secret key sk is discarded and not used in the construction. We note that we only require security of the DPKE scheme for a single message (PRIV1) so the negative result of Wichs [5:3] does not apply. The construction, result and proof are in Section 5.2.

From the LTDF-based DPKE scheme of BFO [15] and LTDFs from [47, 31, 52, 37, 55] we now get IND $\left[\mathbf{X}^{\text {sup }} \cap \mathbf{X}^{\varepsilon} \cap \mathbf{X}^{1}\right]$-secure obfuscators under a large number of standard assumptions. We also get IND $\left[\mathbf{X}^{\text {seup }} \cap \mathbf{X}^{1}\right]$-secure obfuscators under the DLIN, Subgroup Indistinguishability and LWE assumptions via [177, 54, 52]. On the negative side we can rule out PRIV1[ $\left.\mathbf{X}^{\text {cup }}\right]$-secure DPKE under iO via [20, 24].

PO FROM UCE. UCE [6] is a class of assumptions on function families crafted to allow instantiation of random oracles in certain settings. UCE security is parameterized so that we have UCE[S] security of a family of functions for different choices of classes $\mathbf{S}$ of algorithms called sources. The parameterization is necessary because security is not achievable for the class of all sources. Different applications rely on UCE relative to different classes of sources [6, 18, 21, 43., 5, 28].

In this work we use the multi-key version of UCE, abbreviated mUCE [6]. We show how to associate to any given class $\mathbf{X}$ of target generators a class $\mathbf{S}^{\mathbf{X}}$ of sources such that $\mathrm{mUCE}\left[\mathbf{S}^{\mathbf{X}}\right] \Rightarrow$ $\operatorname{IND}[\mathbf{X}]$, meaning we can build a point-function obfuscator secure for $\mathbf{X}$ given a family of functions that is $\operatorname{mUCE}\left[\mathbf{S}^{\mathbf{X}}\right]$-secure. The definition of $\mathbf{S}^{\mathbf{X}}$ is given in Section 5.3. But what is most relevant here is that the strength of UCE-framework assumptions is very sensitive to the choice of class of sources that parameterizes the particular assumption, and $\mathbf{S}^{\mathbf{X}}$ has good properties in this regard. The sources are what are called "split" in [6], and they inherit the unpredictability attributes of the target generators. $\mathrm{mUCE}\left[\mathbf{S}^{\mathbf{X}}\right]$-security is not achievable for all choices of $\mathbf{X}$ but the assumption 
is valid as far as we know for many choices of $\mathbf{X}$, yielding new constructions.

\subsection{Alternative notions and relations between notions}

Above, we fixed one, basic definitional template, which we called IND, and then parameterized it by classes $\mathbf{X}$ of target generators to get notions $\operatorname{IND}[\mathbf{X}]$. However, there are other possible choices for the basic template, some emanating from the literature, and others from the definitional similarity of PO with DPKE. We consider parameterized versions of some of these and relate them to each other and to IND. Specifically we define and consider the following (see Section 6 for formal definitions):

- $\operatorname{SIM}[\mathbf{X}]$ and SSS[X]: (Simulation) Canetti [2:3] defined a security notion for point-function obfuscation called oracle simulatability, which can be thought as a special case (pertaining to point-functions) of the VBB security that was later defined by Barak et al [2]. Definitions of this style were subsequently used in many other papers [41, 51, 3.5, 22.5, 2.9, 14, 42]. With SIM[X] and $\operatorname{SSS}[\mathbf{X}]$ we give $\mathbf{X}$-parameterized versions of the worst-case and average-case simulationbased security definitions, respectively.

- $\operatorname{SIND}[\mathbf{X}]$ : (Strong Indistinguishability) Recall that in $\operatorname{IND}[\mathbf{X}]$, the adversary decision bit is produced as a function of the vector $\overline{\mathbf{P}}$ of obfuscated point functions and the auxiliary information $a$. In $\operatorname{SIND}[\mathbf{X}]$, this bit is not the final decision, but is passed to another adversary who produces the final decision based on it and the target vector itself. This is a parameterized version of the definition of [2:3].

- CSS $[\mathbf{X}]$ : (Comparison-based semantic security) This is an analogue of comparision based semantic security for boolean functions for DPKE [4] in which the adversary needs to compute some predicate on the target vector and auxiliary information.

Fig. $\mathbf{Q}$ shows the relations between five parameterized notions of PO, namely the four above and our original IND $[\mathbf{X}]$.

\subsection{Discussion and further related work}

In concurrent and independent work, BM3 [22] take first steps towards a parameterized definition for point-function obfuscation, with separate definitions for the basic and composable cases. They also show that injective mUCE-secure function families for strongly unpredictable sources making one oracle query per key implies composable AIPO (both for computational and statistical unpredictability), which is a special case of our mUCE result.

Multi-bit auxiliary-input point-function obfuscation (MB-AIPO) [25, 11, 42] allows one to obfuscate the circuit $\mathbf{I}_{k, m}$ that on input $k^{\prime}$ returns $m$ if $k=k^{\prime}$ and $\perp$ otherwise, where $k, m$ are strings. CD [25] show that composable AIPO implies MB-AIPO. MB-AIPO was subsequently used in BP [14] and MH [42]. BM1 [20] show that if $\mathrm{iO}$ is possible then MB-AIPO is not. MB-AIPO seems to be quite a bit stronger than AIPO itself and in particular this result does not rule out AIPO.

In Section 5.$]$ we define $\mathrm{OWF}[\mathbf{X}]$, one-wayness of a function family relative to a class of target generators, the targets here being the inputs to the OWF. We note that $\mathrm{OWF}\left[\mathbf{X}^{\sup } \cap \mathbf{X}^{\varepsilon}\right]$ (inputs are statistically unpredictable and there is no auxiliary information) is the notion of a one-way correlation intractable hash $(\mathrm{CIH})$ function family as per GOR [36].

Our parameterized PRIV1 $[\mathbf{X}]$ notions of security for DPKE schemes apply equally to function families and thus recover, via particular choices of $\mathbf{X}$, some of the security notions for CIH function families from GOR [36]. In these cases, since our DPKE-based constructions of PO do not 
require that decryption in the DPKE scheme is polynomial-time, CIH function families meeting the corresponding notions suffice as well.

A connection that does not seem to have been explicitly made and pursued is that definitionally, there is little to no difference between PO and deterministic public-key encryption (DPKE) [3, 4, 17] or other forms of entropic security [30, 36]. Existing systematic and in-depth consideration of DPKE definitions and relations between them [4, 17] can be exploited to obtain new semantic-security formalizations of PO and also to obtain definitional relations.

\section{Notation and standard definitions}

Notation. We denote by $\lambda \in \mathbb{N}$ the security parameter and by $1^{\lambda}$ its unary representation. We let $\varepsilon$ denote the empty string. If $s$ is an integer then $\operatorname{Pad}_{s}(\mathrm{C})$ denotes circuit $\mathrm{C}$ padded to have size $s$. We say that circuits $\mathrm{C}_{0}, \mathrm{C}_{1}$ are equivalent, written $\mathrm{C}_{0} \equiv \mathrm{C}_{1}$, if they agree on all inputs. If $\mathbf{x}$ is a vector then $|\mathbf{x}|$ denotes the number of its coordinates and $\mathbf{x}[i]$ denotes its $i$-th coordinate. We write $x \in \mathbf{x}$ as shorthand for $x \in\{\mathbf{x}[1], \ldots, \mathbf{x}[|\mathbf{x}|]\}$. If $X$ is a finite set, we let $x \leftarrow \$ X$ denote picking an element of $X$ uniformly at random and assigning it to $x$. Algorithms may be randomized unless otherwise indicated. Running time is worst case. "PT" stands for "polynomial-time," whether for randomized algorithms or deterministic ones. If $A$ is an algorithm, we let $y \leftarrow A\left(x_{1}, \ldots ; r\right)$ denote running $A$ with random coins $r$ on inputs $x_{1}, \ldots$ and assigning the output to $y$. We let $y \leftarrow \$ A\left(x_{1}, \ldots\right)$ be the result of picking $r$ at random and letting $y \leftarrow A\left(x_{1}, \ldots ; r\right)$. We let $\left[A\left(x_{1}, \ldots\right)\right]$ denote the set of all possible outputs of $A$ when invoked with inputs $x_{1}, \ldots$. We say that $f: \mathbb{N} \rightarrow \mathbb{R}$ is negligible if for every positive polynomial $p$, there exists $\lambda_{p} \in \mathbb{N}$ such that $f(\lambda)<1 / p(\lambda)$ for all $\lambda>\lambda_{p}$. We use the code based game playing framework of $[\mathbb{8}]$. (See Fig. 园 for an example.) By $\mathrm{G}^{\mathcal{A}}(\lambda)$ we denote the event that the execution of game $\mathrm{G}$ with adversary $\mathcal{A}$ and security parameter $\lambda$ results in the game returning true.

OBfuscATORS. An obfuscator is a PT algorithm Obf that on input $1^{\lambda}$ and a circuit $\mathrm{C}$ returns a circuit $\overline{\mathrm{C}}$. If $\mathbf{C}$ is an $n$-vector of circuits then $\operatorname{Obf}\left(1^{\lambda}, \mathbf{C}\right)$ denotes the vector $\left(\operatorname{Obf}\left(1^{\lambda}, \mathbf{C}[1]\right), \ldots\right.$, $\left.\operatorname{Obf}\left(1^{\lambda}, \mathbf{C}[n]\right)\right)$ formed by applying Obf independently to each coordinate of $\mathbf{C}$. The correctness condition of obfuscator Obf requires that for every circuit $\mathrm{C}$, every $\lambda \in \mathbb{N}$ and every $\overline{\mathrm{C}} \in\left[\mathrm{Obf}\left(1^{\lambda}, \mathrm{C}\right)\right]$ we have $\overline{\mathrm{C}} \equiv \mathrm{C}$ (meaning $\overline{\mathrm{C}}(x)=\mathrm{C}(x)$ for all $x$ ). We also call the latter a perfect correctness condition and we require that it holds for all obfuscators. We consider various notions of security for obfuscators, namely indistinguishability obfuscation and variants of point-function obfuscation, including AIPO.

INDISTINGUISHABILITY OBFUSCATION. Although our results need only iO, we use diO [2, 16, T] in the proof, applying BCP [16] to then reduce the assumption to iO. To give the definitions compactly, we use the definitional framework of BST [9] which allows us to capture iO variants (including diO) via classes of circuit samplers. Let Obf be an obfuscator. A sampler in this context is a PT algorithm $\mathrm{S}$ that on input $1^{\lambda}$ returns a triple $\left(\mathrm{C}_{0}, \mathrm{C}_{1}\right.$, aux $)$ where $\mathrm{C}_{0}, \mathrm{C}_{1}$ are circuits of the same size, number of inputs and number of outputs, and aux is a string. If $\mathcal{O}$ is an adversary and $\lambda \in \mathbb{N}$ we let $\operatorname{Adv}_{\mathrm{Obf}, \mathrm{S}, \mathcal{O}}^{\text {io }}(\lambda)=2 \operatorname{Pr}\left[\mathrm{IO}_{\mathrm{Obf}, \mathrm{S}}^{\mathcal{O}}(\lambda)\right]-1$ where game $\mathrm{IO}_{\mathrm{Obf}, \mathrm{S}}^{\mathcal{O}}(\lambda)$ is defined in Fig. 四. Now let $\boldsymbol{S}$ be a class (set) of circuit samplers. We say that Obf is $\boldsymbol{S}$-secure if $\operatorname{Adv}_{\mathrm{Obf}, \mathrm{S}, \mathcal{O}}^{\text {(o }}(\cdot)$ is negligible for every PT adversary $\mathcal{O}$ and every circuit sampler $\boldsymbol{S} \in \boldsymbol{S}$. We say that circuit sampler $\mathrm{S}$ produces equivalent circuits if there exists a negligible function $\nu$ such that $\operatorname{Pr}\left[\mathrm{C}_{0} \equiv \mathrm{C}_{1}:\left(\mathrm{C}_{0}, \mathrm{C}_{1}, a u x\right) \leftarrow \$ \mathrm{~S}\left(1^{\lambda}\right)\right] \geq 1-\nu(\lambda)$ for all $\lambda \in \mathbb{N}$. Let $\boldsymbol{S}_{\text {eq }}$ be the class of all circuit samplers that produce equivalent circuits. We say that Obf is an indistinguishability obfuscator if it is $\boldsymbol{S}_{\mathrm{eq}}$-secure [2, 3:3, 4.9]. 


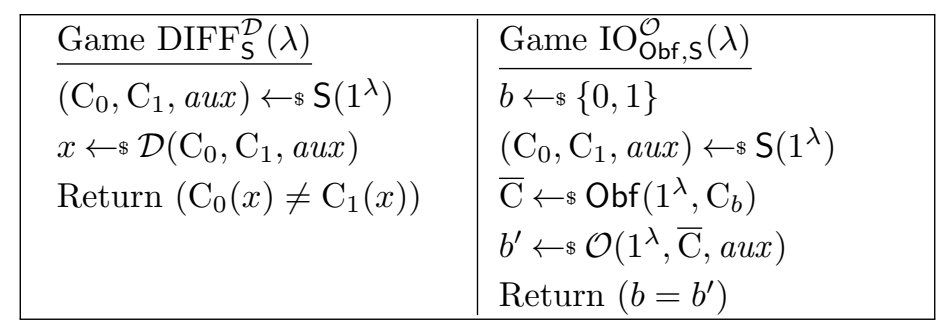

Figure 1: Games defining difference-security of circuit sampler S and iO-security of obfuscator Obf relative to circuit sampler $\mathrm{S}$.

We say that a circuit sampler $S$ is difference secure if $\operatorname{Adv}_{S, \mathcal{D}}^{\text {diff }}(\cdot)$ is negligible for every PT adversary $\mathcal{D}$, where $\operatorname{Adv}_{\mathcal{S}, \mathcal{D}}^{\text {diff }}(\lambda)=\operatorname{Pr}\left[\operatorname{DIFF}_{\mathbf{S}}^{\mathcal{D}}(\lambda)\right]$ and game $\operatorname{DIFF}_{\mathcal{S}}^{\mathcal{D}}(\lambda)$ is defined in Fig. $\mathbb{W}$. Difference security of $\mathrm{S}$ means that given $\mathrm{C}_{0}, \mathrm{C}_{1}$, aux it is hard to find an input on which the circuits differ [ [2, [16, T]. Let $\boldsymbol{S}_{\text {diff }}$ be the class of all difference-secure circuit samplers. We say that circuit sampler $\mathbf{S}$ produces $d$-differing circuits, where $d: \mathbb{N} \rightarrow \mathbb{N}$, if for all $\lambda \in \mathbb{N}$ circuits $\mathrm{C}_{0}$ and $\mathrm{C}_{1}$ differ on at most $d(\lambda)$ inputs with an overwhelming probability over $\left(\mathrm{C}_{0}, \mathrm{C}_{1}\right.$, aux $) \leftarrow \$ \mathrm{~S}\left(1^{\lambda}\right)$. Let $\boldsymbol{S}_{\text {diff }}(d)$ be the class of all difference-secure circuit samplers that produce $d$-differing circuits, so that $\boldsymbol{S}_{\text {eq }} \subseteq \boldsymbol{S}_{\text {diff }}(d) \subseteq$ $\boldsymbol{S}_{\text {diff }}$. The interest of this definition is the following result of BCP [즈] that we use:

Proposition 2.1 If $d$ is a polynomial then any $\boldsymbol{S}_{\mathrm{eq}}$-secure circuit obfuscator is also an $\boldsymbol{S}_{\mathrm{diff}}(d)$ secure circuit obfuscator.

FUnCTION FAMILIES. A family of functions $F$ specifies the following. PT key generation algorithm F.Kg takes $1^{\lambda}$ to return a key $f k \in\{0,1\}^{\mathrm{F} \cdot \mathrm{kl}(\lambda)}$, where $\mathrm{F} . \mathrm{kl}: \mathbb{N} \rightarrow \mathbb{N}$ is the key length function associated to F. Deterministic, PT evaluation algorithm F.Ev takes $1^{\lambda}$, key $f k \in\left[F . \operatorname{Kg}\left(1^{\lambda}\right)\right]$ and an input $x \in\{0,1\}^{\mathrm{F} . \mathrm{il}(\lambda)}$ to return an output $\mathrm{F} . \operatorname{Ev}\left(1^{\lambda}, f k, x\right) \in\{0,1\}^{\mathrm{F} . \mathrm{ol}(\lambda)}$, where F.il, F.ol: $\mathbb{N} \rightarrow \mathbb{N}$ are the input and output length functions associated to $F$, respectively. We say that $F$ is injective if the function $\mathrm{F} . \operatorname{Ev}\left(1^{\lambda}, f k, \cdot\right):\{0,1\}^{\mathrm{F} . \mathrm{il}(\lambda)} \rightarrow\{0,1\}^{\mathrm{F} . \mathrm{ol}(\lambda)}$ is injective for every $\lambda \in \mathbb{N}$ and every $f k \in\left[F . \operatorname{Kg}\left(1^{\lambda}\right)\right]$. Notions of security for function families that we use are mUCE and OWF, the latter defined in Section 5.].

UCE FRAMEWORK. We recall the Universal Computational Extractor (UCE) framework of BHK [6]. We will use what BHK call the multi-key version of UCE (mUCE). It is an extension of the more commonly used UCE notion for a single key, meaning that it implies the latter. Meanwhile, no implications in the other direction (from single-key to multi-key) are known. BM3 [22] propose a multi-key UCE construction in the standard model, whereas BHK [7] and Mittelbach [45] propose constructions in the ROM.

Let $\mathrm{H}$ be a family of functions. Let $\mathcal{S}$ be an adversary called the source and $\mathcal{D}$ an adversary called the distinguisher. Consider game $\operatorname{mUCE}_{\mathrm{H}}^{\mathcal{S}, \mathcal{D}}(\lambda)$ in the left panel of Fig. 2]. Associated to $\mathcal{S}$ is a polynomial $\mathcal{S}$.nk that indicates how many keys $\mathcal{S}$ uses. The source has access to an oracle HASH. A query to HASH consists of an index $i$ of a key and the actual input $x$, which is a string required to have length $\mathrm{H}$.il $(\lambda)$. When the challenge bit $b$ is 1 (the "real" case) the oracle responds via $\mathrm{H}$.Ev under a key $\mathbf{h k}[i]$ that is chosen by the game and not given to the source. When $b=0$ (the "random" case) it responds as a random oracle. The source then leaks a string $L$ to its accomplice distinguisher. The latter does get the key vector hk as input and must now return its guess $b^{\prime} \in\{0,1\}$ for $b$. The game returns true iff $b^{\prime}=b$. The advantage of $(\mathcal{S}, \mathcal{D})$ against the mUCE security of $\mathbf{H}$ is defined for $\lambda \in \mathbb{N}$ via $\operatorname{Adv}_{\mathbf{H}, \mathcal{S}, \mathcal{D}}^{\mathrm{m}}(\lambda)=2 \operatorname{Pr}\left[\operatorname{mUCE}_{\mathrm{H}}^{\mathcal{S}, \mathcal{D}}(\lambda)\right]-1$. If $\mathbf{S}$ is a class 


\begin{tabular}{|c|c|}
\hline $\operatorname{mUCE}_{\mathrm{H}}^{\mathcal{S}, \mathcal{D}}(\lambda)$ & $\underline{\operatorname{mSPRED}} \mathcal{S}_{\mathcal{S}}^{\mathcal{P}}(\lambda)$ \\
\hline For $i=1, \ldots, \mathcal{S} \cdot \operatorname{nk}(\lambda)$ do $\mathbf{h k}[i] \leftarrow{ }_{\$} \mathrm{H} \cdot \mathrm{Kg}\left(1^{\lambda}\right)$ & $X \leftarrow \emptyset ; L \leftarrow{ } \mathcal{S}^{\mathrm{HASH}}\left(1^{\lambda}\right)$ \\
\hline$b \leftarrow ه\{0,1\} ; L \leftarrow \$ \mathcal{S}^{\mathrm{HASH}}\left(1^{\lambda}\right)$ & $x \leftarrow \$ \mathcal{P}\left(1^{\lambda}, L\right)$ \\
\hline$b^{\prime} \leftarrow{ } \mathcal{D}\left(1^{\lambda}, \mathbf{h k}, L\right)$ & Return $(x \in X)$ \\
\hline Return $\left(b=b^{\prime}\right)$ & $\operatorname{HASH}(i, x)$ \\
\hline$\underline{\operatorname{HASH}(i, x)}$ & If not $(1 \leq i \leq \mathcal{S} \cdot \operatorname{nk}(\lambda))$ then \\
\hline If not $(1 \leq i \leq \mathcal{S}$.nk $(\lambda))$ then return $\perp$ & Return $\perp$ \\
\hline If $T[i, x]=\perp$ then & If $T[i, x]=\perp$ then \\
\hline If $b=0$ then $T[i, x] \leftarrow \$\{0,1\}^{\mathrm{H} . \mathrm{ol}(\lambda)}$ & $T[i, x] \leftarrow ه\{0,1\}^{\mathrm{H} . \mathrm{ol}(\lambda)}$ \\
\hline Else $T[i, x] \leftarrow \operatorname{H.Ev}\left(1^{\lambda}, \mathbf{h k}[i], x\right)$ & $X \leftarrow X \cup\{x\}$ \\
\hline Return $T[i, x]$ & Return $T[i, x]$ \\
\hline
\end{tabular}

Figure 2: Games defining mUCE security of function family $\mathrm{H}$ and unpredictability of source $\mathcal{S}$.

(set) of sources, we say that $\mathrm{H}$ is $\operatorname{mUCE}[\mathbf{S}]$-secure if $\operatorname{Adv}_{\mathrm{H}, \mathcal{S}, \mathcal{D}}^{\mathrm{m}}(\cdot)$ is negligible for all sources $\mathcal{S} \in \mathbf{S}$ and all PT distinguishers $\mathcal{D}$.

It is easy to see that $\mathrm{mUCE}[\mathbf{S}]$-security is not achievable if $\mathbf{S}$ is the class of all PT sources [6]. To obtain meaningful notions of security, BHK [6] impose restrictions on the source. A central restriction is unpredictability. A source is unpredictable if it is hard to guess the source's HASH queries even given the leakage, in the random case of the mUCE game. Formally, let $\mathcal{S}$ be a source and $\mathcal{P}$ an adversary called a predictor and consider game $\operatorname{mSPRED}_{\mathcal{S}}^{\mathcal{P}}(\lambda)$ in Fig. 2 . For $\lambda \in \mathbb{N}$ we let $\operatorname{Adv}_{\mathcal{S}, \mathcal{P}}^{\mathrm{m} \text {-spred }}(\lambda)=\operatorname{Pr}\left[\operatorname{mSPRED}_{\mathcal{S}}^{\mathcal{P}}(\lambda)\right]$. We say that $\mathcal{S}$ is computationally unpredictable if $\operatorname{Adv}_{\mathcal{S}, \mathcal{P}}^{\mathrm{m} \text {-spred }}(\cdot)$ is negligible for all PT predictors $\mathcal{P}$, and let $\mathbf{S}^{\text {cup }}$ be the class of all PT computationally unpredictable sources. We say that $\mathcal{S}$ is statistically unpredictable if $\operatorname{Adv}_{\mathcal{S}, \mathcal{P}}^{\mathrm{m} \text {-spred }}(\cdot)$ is negligible for all (not necessarily PT) predictors $\mathcal{P}$, and let $\mathbf{S}^{\text {sup }} \subseteq \mathbf{S}^{\text {cup }}$ be the class of all PT statistically unpredictable sources. We say that $\mathcal{S}$ is sub-exponentially unpredictable if there is an $\epsilon>0$ such that for any PT predictor $\mathcal{P}$ there is a $\lambda_{\mathcal{P}}$ such that $\operatorname{Adv}_{\mathcal{S}, \mathcal{P}}^{\mathrm{m} \text {-sped }}(\lambda) \leq 2^{-\lambda^{\epsilon}}$ for all $\lambda \geq \lambda_{\mathcal{P}}$, and let $\mathbf{S}^{\text {seup }} \subseteq \mathbf{S}^{\text {cup }}$ be the class of all PT sub-exponentially unpredictable sources.

BFM [18] show that UCE-framework security notions (both single-key and multi-key) are not achievable for $\mathbf{S}^{\text {cup }}$ assuming that indistinguishability obfuscation exists. This has lead applications to impose further restrictions on the source by using either $\mathbf{S}^{\text {sup }}$ or subsets of $\mathbf{S}^{\text {cup }}$. Assumptions based on $\mathbf{S}^{\text {sup }}$, introduced in [6, [8], at this point seem to be viable. In order to restrict the computational case, one can consider split sources as defined in BHK [6]. Such sources can leak information about oracle queries and answers separately, but not together. We let $\mathbf{S}^{\text {splt }}$ denote the class of split sources. Another way to restrict a source is by limiting the number of queries it can make. Let $\mathbf{S}^{n, q}$ be the class of sources $\mathcal{S}$ such that $\mathcal{S}$.nk $(\cdot) \leq n(\cdot)$ and $\mathcal{S}$ makes at most $q(\cdot)$ queries to each key. In particular $\mathbf{S}^{1,1}$ is the class of sources that use only one key and make only one query to it.

\section{Point-function obfuscation framework}

The literature considers many different variants of point function obfuscation. Here we provide a definitional framework that unifies these concepts and allows us to obtain not just known but also new variants of point function obfuscation as special cases. The framework parameterizes the 


\begin{tabular}{|c|c|c|}
\hline Game $\operatorname{IND}_{\mathrm{Obf}, \mathrm{X}}^{\mathcal{A}}(\lambda)$ & $\operatorname{Game~}_{\operatorname{PRED}}^{\mathcal{Q}}(\lambda)$ & ${\operatorname{Game~} \operatorname{TRIV}_{\mathrm{X}}^{\mathcal{A}}(\lambda)}$ \\
\hline$b \leftarrow \$\{0,1\}$ & $(\mathbf{k}, a) \leftarrow \$ \operatorname{X} \cdot \operatorname{Ev}\left(1^{\lambda}\right)$ & $b \leftarrow s\{0,1\}$ \\
\hline$\left(\mathbf{k}_{1}, a_{1}\right) \leftarrow s \mathrm{X} \cdot \operatorname{Ev}\left(1^{\lambda}\right)$ & $k \leftarrow \mathcal{Q}\left(1^{\lambda}, a\right)$ & $\left(\mathbf{k}_{1}, a_{1}\right) \leftarrow \$ \operatorname{X} \cdot \operatorname{Ev}\left(1^{\lambda}\right)$ \\
\hline $\begin{array}{l}\text { For } i=1, \ldots, \mathrm{X} \cdot \mathrm{vl}(\lambda) \text { do } \\
\mathbf{k}_{0}[i] \leftarrow \$\{0,1\}^{\mathrm{X} \cdot \mathrm{tl}(\lambda)}\end{array}$ & $\operatorname{Return}(\exists i: \mathbf{k}[i]=k)$ & $\begin{array}{c}\text { For } i=1, \ldots, \mathrm{X} \cdot \mathrm{vl}(\lambda) \mathrm{do} \\
\mathbf{k}_{0}[i] \leftarrow \$\{0,1\}^{\mathrm{X} \cdot \mathrm{tl}(\lambda)}\end{array}$ \\
\hline$\overline{\mathbf{P}} \leftarrow \$ \operatorname{Obf}\left(1^{\lambda}, \mathbf{I}_{\mathbf{k}_{b}}\right)$ & & $b^{\prime} \leftarrow \Phi \mathcal{A}\left(1^{\lambda}, \mathbf{k}_{b}, a_{1}\right)$ \\
\hline$b^{\prime} \leftarrow \Phi \mathcal{A}\left(1^{\lambda}, \overline{\mathbf{P}}, a_{1}\right)$ & & Return $\left(b=b^{\prime}\right)$ \\
\hline $\operatorname{Return}\left(b=b^{\prime}\right)$ & & \\
\hline
\end{tabular}

Figure 3: Games defining IND security of point-function obfuscator Obf relative to target generator $\mathbf{X}$, unpredictabilty of target generator $\mathbf{X}$ and triviality of target generator $\mathbf{X}$.

security of a point-obfuscator by a class of algorithms we call target generators. Different notions of point obfuscation then correspond to different choices of this class. We start by defining target generators.

TARget Generators. A target generator $\mathrm{X}$ specifies a PT algorithm $\mathrm{X}$.Ev that takes $1^{\lambda}$ to return a target vector $\mathbf{k}$ and auxiliary information $a \in\{0,1\}^{*}$. The entries of $\mathbf{k}$ are the targets, each of length $\mathrm{X} . \mathrm{tl}(\lambda)$, and the vector itself has length $\mathrm{X} . \mathrm{vl}(\lambda)$, where $\mathrm{X} . \mathrm{tl}, \mathrm{X} . \mathrm{vl}: \mathbb{N} \rightarrow \mathbb{N}$ are the target length and target-vector length functions associated to $X$, respectively.

Point-Function obfuscation. If $k$ is a bit-string then $\mathbf{I}_{k}:\{0,1\}^{|k|} \rightarrow\{0,1\}$ denotes a canonical representation of the circuit that on input $k^{\prime} \in\{0,1\}^{|k|}$ returns 1 if $k=k^{\prime}$ and 0 otherwise. It is assumed that given $\mathbf{I}_{k}$, one can compute $k$ in time linear in $|k|$. A circuit $\mathrm{C}$ is called a point circuit if there is a $k$, called the circuit target, such that $\mathrm{C} \equiv \mathbf{I}_{k}$. If $\mathbf{k}$ is an $n$-vector of strings then we let $\mathbf{I}_{\mathbf{k}}=\left(\mathbf{I}_{\mathbf{k}[1]}, \ldots, \mathbf{I}_{\mathbf{k}[n]}\right)$.

Let Obf be an obfuscator, as defined in Section [2. Its correctness condition guarantees that on input $1^{\lambda}, \mathbf{I}_{k}$, it returns a point circuit with target $k$, which is the condition for calling it a pointfunction obfuscator. We say that Obf has target length Obf.tl: $\mathbb{N} \rightarrow \mathbb{N}$ if its correctness condition is only required on inputs $\mathbf{I}_{k}$ with $k \in\{0,1\}^{\mathrm{Obf.t}(\lambda)}$.

SECURITY OF POINT-FUnCTION OBFUSCATION. We now define security of point-function obfuscator relative to a class of target generators. We will then consider various choices of these classes.

Consider game IND of Fig. 3 associated to a point-function obfuscator Obf, a target generator $\mathrm{X}$ and an adversary $\mathcal{A}$, such that Obf.tl $=\mathrm{X}$.tl. For $\lambda \in \mathbb{N}$ let $\operatorname{Adv}_{\mathrm{Obf}, \mathrm{X}, \mathcal{A}}^{\mathrm{ind}}(\lambda)=2 \operatorname{Pr}\left[\operatorname{IND}_{\mathrm{Obf}, \mathrm{X}}^{\mathcal{A}}(\lambda)\right]-1$. The game generates a target vector $\mathbf{k}_{1}$ and corresponding auxiliary information $a_{1}$ via $X$. It also samples a target vector $\mathbf{k}_{0}$ uniformly at random, containing $\mathrm{X} \cdot \mathrm{vl}(\lambda)$ elements each of length $\mathrm{X} \cdot \mathrm{tl}(\lambda)$. It then obfuscates the targets in the challenge vector $\mathbf{k}_{b}$ via Obf to produce $\overline{\mathbf{P}}$ which, as per our notation, will be the vector $\left(\operatorname{Obf}\left(1^{\lambda}, \mathbf{I}_{\mathbf{k}_{b}[1]}\right), \ldots, \operatorname{Obf}\left(1^{\lambda}, \mathbf{I}_{\mathbf{k}_{b}[\mathbf{X} \cdot \mathbf{v l}(\lambda)]}\right)\right)$ formed by independently obfuscating the targets in the target vector. Given $\overline{\mathbf{P}}$ and $a_{1}$, adversary $\mathcal{A}$ outputs a bit $b^{\prime}$, and wins the game if this equals $b$, meaning it guesses whether the target vector that was obfuscated was the one corresponding to auxiliary information $a_{1}$ or one independent of it.

Let $\mathbf{X}$ be a class (set) of target generators. We say that $\operatorname{Obf}$ is $\operatorname{IND}[\mathbf{X}]$-secure if $\operatorname{Adv}_{\mathrm{Obf}, \mathbf{X}, \mathcal{A}}^{\text {ind }}(\cdot)$ is negligible for every $\mathrm{PT} \mathcal{A}$ and every $\mathbf{X} \in \mathbf{X}$. We now capture different notions in the literature, as well as new ones, by considering particular classes $\mathbf{X}$. At the end of this section we will present what we call the triviality theorem, showing how the definition is vacuous for some classes, and discuss its implications. We will further discuss alternative security definitions for point-function obfuscation in Section [6]. 
Classes of TARGET GENERATORS. One important (and necessary) condition on a target generator is unpredictability. To define this, consider game PRED of Fig. 3 associated to $\mathbf{X}$ and a predictor adversary $\mathcal{Q}$. For $\lambda \in \mathbb{N}$ let $\operatorname{Adv}_{\mathrm{X}, \mathcal{Q}}^{\text {pred }}(\lambda)=\operatorname{Pr}\left[\operatorname{PRED}_{\mathbf{X}}^{\mathcal{Q}}(\lambda)\right]$. The game generates a target vector $\mathbf{k}$ and associated auxiliary information $a$. The adversary $\mathcal{Q}$ gets $a$ and wins if it can predict any entry of the vector $\mathbf{k}$.

The first dimension along which point-function obfuscators are classified is the type of unpredictability, encompassing two sub-dimensions: the success probability of predictors (may be required to be negligible or sub-exponential) and their computational power (PT and computationally unbounded are the popular choices, but one could also consider sub-exponential time). Some relevant classes are the following:

- $\mathbf{X}^{\text {cup }}$ - Class of computationally unpredictable target generators $-\mathbf{X} \in \mathbf{X}^{\text {cup }}$ if $\operatorname{Adv}_{\mathbf{X}, \mathcal{Q}}^{\text {pred }}(\cdot)$ is negligible for all PT predictor adversaries $\mathcal{Q}$.

- $\mathbf{X}^{\text {seup }}$ - Class of sub-exponentially unpredictable target generators $-\mathbf{X} \in \mathbf{X}^{\text {seup }}$ if there exists $0<\epsilon<1$ such that for every PT predictor adversary $\mathcal{Q}$ there is a $\lambda_{\mathcal{Q}}$ such that $\operatorname{Adv}_{\mathrm{X}, \mathcal{Q}}^{\text {pred }}(\lambda) \leq 2^{-\lambda^{\epsilon}}$ for all $\lambda \geq \lambda_{\mathcal{Q}}$.

- $\mathbf{X}^{\text {sup }}$ - Class of statistically unpredictable target generators $-\mathbf{X} \in \mathbf{X}^{\text {sup }}$ if $\operatorname{Adv}_{\mathbf{X}, \mathcal{Q}}^{\text {pred }}(\cdot)$ is negligible for all (even computationally unbounded) predictor adversaries $\mathcal{Q}$.

Another dimension is the number of target points in the target vector, to capture which, for any polynomial $q: \mathbb{N} \rightarrow \mathbb{N}$, we let

- $\mathbf{X}^{q(\cdot)}$ — Class of generators producing $q(\cdot)$ target points $-\mathbf{X} \in \mathbf{X}^{q(\cdot)}$ if $\mathbf{X} \cdot \mathbf{v l}=q$. An important special case is $q(\cdot)=1$.

Another important dimension is auxiliary information, which may be present or absent (the latter, formally means it is the empty string), to capture which we let

- $\mathbf{X}^{\varepsilon}$ - Class of generators with no auxiliary information $-\mathbf{X} \in \mathbf{X}^{\varepsilon}$ if $a=\varepsilon$ for all $(\mathbf{k}, a) \in$ $\left[\mathrm{X} . \operatorname{Ev}\left(1^{\lambda}\right)\right]$ and all $\lambda \in \mathbb{N}$.

We can recover notions from the literature as follows:

- $\operatorname{IND}\left[\mathbf{X}^{\text {cup }} \cap \mathbf{X}^{\varepsilon} \cap \mathbf{X}^{1}\right]$ - This is basic point-function obfuscation, secure for a single computationally unpredictable target point, and no auxiliary information is allowed. It is achieved in [2:3, [27, 41, 51].

- $\operatorname{IND}\left[\mathbf{X}^{\text {cup }} \cap \mathbf{X}^{1}\right]$ — This is AIPO [2:3, [3.5, 14, 20], secure for a single computationally unpredictable target point in the presence of auxiliary information. It is achieved under the AI-DHI assumption by Canetti [2:3], and using the extended construction of Wee [5] by BP [14].

- $\operatorname{IND}\left[\mathbf{X}^{\text {cup }}\right]$ - This is composable AIPO [25, 14], meaning that it is secure for arbitrarily many correlated target points that are computationally unpredictable in the presence of auxiliary information. BM1 [20] showed that this notion cannot co-exit with $\mathrm{iO}$ in the presence of OWFs.

- $\operatorname{IND}\left[\mathbf{X}^{\sup } \cap \mathbf{X}^{\varepsilon}\right]$ - This is composable point-function obfuscation with no auxiliary information allowed [41, 42], secure for arbitrarily many correlated target points that are statistically unpredictable. It is achieved from $\mathrm{mUCE}\left[\mathbf{S}^{\text {sup }}\right]$ in BHK [6] .

Furthermore, DKL [2.9] achieve $\operatorname{IND}\left[\mathbf{X}^{\text {sup }} \cap \mathbf{X}^{1}\right]$ from the LSN (i.e. auxiliary-input LPN) assumption and BM3 [22] build IND $\left[\mathbf{X}^{\text {sup }}\right]$ from $\mathrm{mUCE}\left[\mathbf{S}^{\mathbf{s}-\sup } \cap \mathbf{X}^{1}\right]$. Here $\mathbf{S}^{\mathrm{s} \text {-sup }}$ is used to denote sources with "strong statistical unpredictability" as defined in BM2 [21], who also showed that $\mathbf{S}^{\mathbf{s}-\text { sup }}$ is a strictly larger class than $\mathbf{S}^{\text {sup }} \cap \mathbf{S}^{\text {splt }}$. We note that some of the above results achieve notions that are stronger than IND. Such notions are discussed and defined in Section 6 . 


\begin{tabular}{|c|c|}
\hline $\begin{array}{l}\text { Game } \operatorname{MDIFF}_{\mathrm{S}}^{\mathcal{D}}(\lambda) \\
\left(\mathbf{C}_{0}, \mathbf{C}_{1}, \text { aux }\right) \leftarrow \$ \mathrm{~S}\left(1^{\lambda}\right) \\
x \leftarrow{ }^{\lambda}\left(\mathbf{C}_{0}, \mathbf{C}_{1}, \text { aux }\right) \\
\operatorname{Return}\left(\exists i: \mathbf{C}_{0}[i](x) \neq \mathbf{C}_{1}[i](x)\right)\end{array}$ & $\begin{array}{l}\text { Game } \mathrm{MIO}_{\mathrm{Obf}, \mathrm{S}}^{\mathcal{O}}(\lambda) \\
b \leftarrow \$\{0,1\} \\
\left(\mathbf{C}_{0}, \mathbf{C}_{1}, \text { aux }\right) \leftarrow \$ \mathrm{~S}\left(1^{\lambda}\right) \\
\overline{\mathbf{C}} \leftarrow_{\S} \operatorname{Obf}\left(1^{\lambda}, \mathbf{C}_{b}\right) \\
b^{\prime} \leftarrow \Phi \mathcal{O}\left(1^{\lambda}, \overline{\mathbf{C}}, \text { aux }\right) \\
\operatorname{Return}\left(b=b^{\prime}\right)\end{array}$ \\
\hline
\end{tabular}

Figure 4: Games defining difference-security of multi-circuit sampler S and iO-security of obfuscator Obf relative to multi-circuit sampler $\mathrm{S}$.

Triviality TheOREM. The IND $[\mathbf{X}]$ definition has the peculiar property of trivializing for some choices of $\mathbf{X}$. For example, let $\mathbf{X}$ be a target generator that returns a vector of random, independent targets and auxiliary information $a=\varepsilon$ the empty string. Then any point-function obfuscator Obf is $\operatorname{IND}[\{\mathbf{X}\}]$-secure. This is true because game IND in this case samples $\mathbf{k}_{0}, \mathbf{k}_{1}$ from the same distribution and the information provided to the adversary $\mathcal{A}$ is thus independent of the challenge bit. Before discussing and assessing what this means for the definition, we provide a general triviality theorem that characterizes for what choices of $\mathbf{X}$ this phenomenon happens.

Consider game TRIV of Fig. [3 associated to a target generator $\mathrm{X}$ and an adversary $\mathcal{A}$. For $\lambda \in \mathbb{N}$ let $\operatorname{Adv}_{\mathrm{X}, \mathcal{A}}^{\text {triv }}(\lambda)=2 \operatorname{Pr}\left[\operatorname{TRIV}_{\mathrm{X}}^{\mathcal{A}}(\lambda)\right]-1$. We say that $\mathrm{X}$ is trivial if $\operatorname{Adv}_{\mathrm{X}, \mathcal{A}}^{\text {triv }}(\cdot)$ is negligible for every $\mathrm{PT} \mathcal{A}$. An example of trivial $\mathrm{X}$ is the one given above. Let $\mathbf{X}^{\text {triv }}$ be the class of all trivial target generators, and say that a class $\mathbf{X}$ is trivial if $\mathbf{X} \subseteq \mathbf{X}^{\text {triv }}$. The proof of the following triviality theorem follows directly from the definitions of games IND and TRIV and is omitted.

Theorem 3.1 Let $\mathbf{X} \subseteq \mathbf{X}^{\text {triv }}$ be a class of target generators. Let Obf be any point-function obfuscator. Then $\mathrm{Obf}$ is IND $[\mathbf{X}]$-secure.

This can be viewed as a defect of the IND definition, but whether or not this is true is debatable. The IND definition has been successfully employed in applications [14, 21]. In these cases, $\mathbf{X}=\mathbf{X}^{\text {cup }} \cap$ $\mathbf{X}^{1}$, a class to which Theorem $\mathbf{3 . 0}$ does not apply. This indicates that the classes of target generators arising in applications are naturally not trivial. And the constructions we give in Section 5 cover such non-trivial classes. Thus we are on the whole unsure whether or not Theorem [.] should be viewed as a definitional weakness. In Section [6 we will provide alternative security definitions for PO that avoid this type of triviality theorem and are meaningful for all choices of target generators. But if an application can be obtained via IND, then it seems preferable, since this definition is simpler and easier to use and, from Section 5 , we have more constructions for it.

\section{4 (d)iO for multi-circuit samplers}

We state and prove a lemma we will use that may be of independent interest. We extend the standard definition of circuit samplers from Section $\nabla$ to get multi-circuit samplers, which are samplers that may produce a vector of circuit pairs (but still only a single auxiliary information string). We also extend the security definition of differing-inputs obfuscation to work with respect to multi-circuit samplers. We then use a hybrid argument to show that the security of the latter is implied by the standard definition of differing-inputs obfuscation for circuit samplers that produce only a single pair of circuits. This result will be used for our iO-based construction of a pointfunction obfuscator, BCP [16] being applied to move from diO to iO. (We stress that diO is used as a tool but not as an assumption in our results.) 


$\frac{\text { Game } \mathrm{G}_{\ell}}{\left(\mathbf{C}_{0}, \mathbf{C}_{1}, a u x^{\mathrm{m}}\right) \leftarrow{ }^{\mathrm{m}} \mathrm{S}^{\mathrm{m}}\left(1^{\lambda}\right) ; n \leftarrow \operatorname{S.vl}(\lambda)}$
For $i=1, \ldots, \ell$ do $\overline{\mathbf{C}}[i] \leftarrow_{\$} \operatorname{Obf}\left(1^{\lambda}, \mathbf{C}_{0}[i]\right)$
For $i=\ell+1, \ldots, n$ do $\overline{\mathbf{C}}[i] \leftarrow{ }_{\$} \operatorname{Obf}\left(1^{\lambda}, \mathbf{C}_{1}[i]\right)$
$b^{\prime} \leftarrow \mathcal{O}^{\mathrm{m}}\left(1^{\lambda}, \mathbf{C}\right.$, aux $\left.^{\mathrm{m}}\right) ; \operatorname{Return}\left(b^{\prime}=1\right)$

Figure 5: Games for proof of Lemma 4.1.

IO FOR MULTI-CIRCUIT SAMPLERS. A multi-circuit sampler is a PT algorithm $\mathrm{S}$ with an associated circuit-vector length function $\mathbf{S} . v 1: \mathbb{N} \rightarrow \mathbb{N}$. Algorithm $\mathbf{S}$ on input $1^{\lambda}$ returns a triple $\left(\mathbf{C}_{0}, \mathbf{C}_{1}\right.$, aux $)$ where aux is a string and $\mathbf{C}_{0}, \mathbf{C}_{1}$ are circuit vectors of length $\mathbf{S} \cdot \mathrm{vl}(\lambda)$, such that circuits $\mathbf{C}_{0}[i]$ and $\mathbf{C}_{1}[i]$ are of the same size, number of inputs and number of outputs for every $i \in\{1, \ldots, \operatorname{S} . v \mathrm{l}(\lambda)\}$.

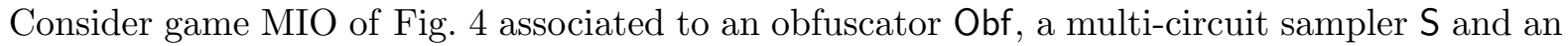
adversary $\mathcal{O}$. For $\lambda \in \mathbb{N}$ let $\operatorname{Adv}_{\text {Obf }, S, \mathcal{O}}^{\text {mio }}(\lambda)=2 \operatorname{Pr}\left[\operatorname{MIO}_{\text {Obf }, \mathcal{S}}^{\mathcal{O}}(\lambda)\right]-1$. Let $\boldsymbol{S}$ be a class of multi-circuit samplers. We say that $\mathrm{Obf}$ is $\boldsymbol{S}$-secure if $\operatorname{Adv}_{\mathrm{Obf}, \mathrm{S}, \mathcal{O}}^{\mathrm{m}}(\cdot)$ is negligible for every multi-circuit sampler $\boldsymbol{S} \in \boldsymbol{S}$ and every PT adversary $\mathcal{O}$.

Consider game MDIFF of Fig. $\mathbb{\text { ⿴囗十 }}$ associated to a multi-circuit sampler $S$ and an adversary $\mathcal{D}$. For $\lambda \in \mathbb{N}$ let $\operatorname{Adv}_{\mathrm{S}, \mathcal{D}}^{\text {m-diff }}(\lambda)=\operatorname{Pr}\left[\operatorname{MDIFF}_{\mathrm{S}}^{\mathcal{D}}(\lambda)\right]$. We say that a multi-circuit sampler $\mathrm{S}$ is difference secure if $\operatorname{Adv}_{\mathrm{S}, \mathcal{D}}^{\mathrm{m} \text {-diff }}(\cdot)$ is negligible for every PT adversary $\mathcal{D}$. Let $\boldsymbol{S}_{\mathrm{m} \text {-diff }}$ be the class of all difference-secure multi-circuit samplers and let $d: \mathbb{N} \rightarrow \mathbb{N}$. We say that multi-circuit sampler $\mathrm{S}$ produces $d$-differing circuits if circuits $\mathrm{C}_{0}[i]$ and $\mathrm{C}_{1}[i]$ differ on at most $d(\lambda)$ inputs with an overwhelming probability over $\left(\mathbf{C}_{0}, \mathbf{C}_{1}, a u x\right) \in\left[\mathbf{S}\left(1^{\lambda}\right)\right]$, for all $\lambda \in \mathbb{N}$ and all $i \in\{1, \ldots, \mathbf{S} \cdot v \mathbf{v l}(\lambda)\}$. Let $\boldsymbol{S}_{\mathrm{m} \text {-diff }}(d)$ be the class of all difference-secure multi-circuit samplers that produce $d$-differing circuits.

Lemma 4.1 Let $d: \mathbb{N} \rightarrow \mathbb{N}$. Let Obf be an $\boldsymbol{S}_{\text {diff }}(d)$-secure obfuscator. Then Obf is also an $\boldsymbol{S}_{\mathrm{m} \text {-diff }}(d)$-secure obfuscator.

Proof of Lemma 4.T: Let $\mathrm{S}^{\mathrm{m}} \in \boldsymbol{S}_{\text {m-diff }}(d)$ be a multi-circuit sampler. Let $\mathcal{O}^{\mathrm{m}}$ be a PT adversary. Let $\lambda \in \mathbb{N}$ and $n=\mathrm{S} \cdot \mathrm{vl}(\lambda)$. Consider the games $\mathrm{G}_{\ell}$ of Fig. 1 for $\ell \in\{0, \ldots, n\}$. By construction, we have

$$
\operatorname{Pr}\left[\mathrm{G}_{0}\right]=\operatorname{Pr}\left[\mathrm{MIO}_{\mathrm{Obf}, \mathrm{S}^{\mathrm{m}}}^{\mathcal{O}^{\mathrm{m}}}(\lambda) \mid b=1\right] \text { and } \operatorname{Pr}\left[\mathrm{G}_{n}\right]=\operatorname{Pr}\left[\mathrm{MIO}_{\mathrm{Obf}, \mathrm{S}^{\mathrm{m}}}^{\mathcal{O}^{\mathrm{m}}}(\lambda) \mid b=0\right] .
$$

It follows that $\operatorname{Adv}_{\text {Obf }, S^{\mathrm{m}}, \mathcal{O}_{\mathrm{m}}^{\mathrm{m}}}^{\mathrm{m}}(\lambda)=\operatorname{Pr}\left[\mathrm{G}_{0}\right]-\operatorname{Pr}\left[\mathrm{G}_{n}\right]=\sum_{i=1}^{n}\left(\operatorname{Pr}\left[\mathrm{G}_{i-1}\right]-\operatorname{Pr}\left[\mathrm{G}_{i}\right]\right)$. We will show that this sum is bounded by a negligible function, therefore proving the lemma. We construct a circuit sampler $\mathrm{S}^{\mathrm{s}}$ and a PT adversary $\mathcal{O}^{\text {s }}$ as follows:

$$
\begin{aligned}
& \text { Circuit Sampler } \mathrm{S}^{\mathrm{s}}\left(1^{\lambda}\right) \\
& n \leftarrow \mathrm{S} . \mathrm{vl}(\lambda) ; \ell \leftarrow \$\{1, \ldots, n\} \\
& \left(\mathbf{C}_{0}, \mathbf{C}_{1}, a u x^{\mathrm{m}}\right) \leftarrow \$ \mathrm{~S}^{\mathrm{m}}\left(1^{\lambda}\right) \\
& \text { For } i=1, \ldots, \ell-1 \text { do } \overline{\mathbf{C}}[i] \leftarrow \$ \operatorname{Obf}\left(1^{\lambda}, \mathbf{C}_{0}[i]\right) \\
& \text { For } i=\ell+1, \ldots, n \text { do } \overline{\mathbf{C}}[i] \leftarrow \$ \operatorname{Obf}\left(1^{\lambda}, \mathbf{C}_{1}[i]\right) \\
& \overline{\mathbf{C}}_{l} \leftarrow \overline{\mathbf{C}}[1], \ldots, \overline{\mathbf{C}}[\ell-1] ; \overline{\mathbf{C}}_{r} \leftarrow \overline{\mathbf{C}}[\ell+1], \ldots, \overline{\mathbf{C}}[n] \\
& e \leftarrow \$\{0,1\} ; \text { aux }^{\mathrm{s}} \leftarrow\left(\overline{\mathbf{C}}_{l}, \overline{\mathbf{C}}_{r}, e, a u x^{\mathrm{m}}\right) \\
& \text { Return }\left(\mathbf{C}_{0}[\ell], \mathbf{C}_{1}[\ell], a u x^{\mathrm{s}}\right) \\
& \frac{\text { Adversary } \mathcal{O}^{\mathrm{s}}\left(1^{\lambda}, \overline{\mathrm{C}}, a u x^{\mathrm{s}}\right)}{\left(\overline{\mathbf{C}}_{l}, \overline{\mathbf{C}}_{r}, e, a u x^{\mathrm{m}}\right) \leftarrow a u x^{\mathrm{s}}} \\
& \overline{\mathbf{C}} \leftarrow\left(\overline{\mathbf{C}}_{l}, \overline{\mathrm{C}}, \overline{\mathbf{C}}_{r}\right) \\
& e^{\prime} \leftarrow \$ \mathcal{O}^{\mathrm{m}}\left(1^{\lambda}, \overline{\mathbf{C}}, a u x^{\mathrm{m}}\right) \\
& \text { If }\left(e=e^{\prime}\right) \text { then return } 1 \\
& \text { Else return } 0
\end{aligned}
$$

Let $\ell$ be the value sampled by $\mathrm{S}^{\mathrm{s}}$ in game $\mathrm{IO}_{\mathrm{Obf}, \mathrm{S}^{\mathrm{s}}}^{\mathcal{O}^{\mathrm{s}}}(\lambda)$. For any $i \in\{1, \ldots, n\}$ we have $\operatorname{Pr}\left[\mathrm{G}_{i-1}\right]-$ $\operatorname{Pr}\left[\mathrm{G}_{i}\right]=\operatorname{Pr}\left[\operatorname{Adv}_{\mathrm{Obf}, \mathcal{S}^{\mathrm{s}}, \mathcal{O}_{\mathrm{s}}^{\mathrm{s}}}^{\mathrm{io}}(\lambda): \ell=i\right]$, and hence $\sum_{i=1}^{n}\left(\operatorname{Pr}\left[\mathrm{G}_{i-1}\right]-\operatorname{Pr}\left[\mathrm{G}_{i}\right]\right)=n \cdot \operatorname{Adv}_{\mathrm{Obf}, \mathrm{S}^{\mathrm{s}}, \mathcal{O}^{\mathrm{s}}}^{\mathrm{io}}(\lambda)$. 


\begin{tabular}{|c|c|}
\hline Game $\operatorname{OWF}_{\mathrm{F}, \mathrm{X}}^{\mathcal{F}}(\lambda)$ & Game PRIV1 $1_{\mathrm{DPKE}, \mathrm{X}}^{\mathcal{A}}(\lambda)$ \\
\hline$(\mathbf{k}, a) \leftarrow \$ \mathrm{X} \cdot \operatorname{Ev}\left(1^{\lambda}\right)$ & $b \leftarrow \$\{0,1\} ;\left(\mathbf{k}_{1}, a\right) \leftarrow \AA \mathrm{X} \cdot \operatorname{Ev}\left(1^{\lambda}\right)$ \\
\hline $\begin{array}{l}\text { For } i=1, \ldots, \mathrm{X} \cdot \mathrm{vl}(\lambda) \text { do } \\
\quad \mathbf{f k}[i] \leftarrow{ }_{s} \mathrm{~F} \cdot \operatorname{Kg}\left(1^{\lambda}\right)\end{array}$ & $\begin{array}{l}\text { For } i=1, \ldots, \mathrm{X} \cdot \mathrm{vl}(\lambda) \text { do } \\
\quad \mathbf{k}_{0}[i] \leftarrow \$\{0,1\}^{\mathrm{DPKE} \cdot \mathrm{ml}(\lambda)}\end{array}$ \\
\hline $\mathbf{y}[i] \leftarrow \mathrm{F} . \operatorname{Ev}\left(1^{\lambda}, \mathbf{f k}[i], \mathbf{k}[i]\right)$ & $(\mathbf{p k}[i], \mathbf{s k}[i]) \leftarrow s$ DPKE. Kg $\left(1^{\lambda}\right)$ \\
\hline$k \leftarrow \$ \mathcal{F}\left(1^{\lambda}, \mathbf{f k}, \mathbf{y}, a\right)$ & $\mathbf{c}[i] \leftarrow \operatorname{DPKE} . \operatorname{Enc}\left(1^{\lambda}, \mathbf{p k}[i], \mathbf{k}_{b}[i]\right)$ \\
\hline $\operatorname{Return}\left(\exists i: \operatorname{F} \cdot \operatorname{Ev}\left(1^{\lambda}, \mathbf{f k}[i], k\right)=\mathbf{y}[i]\right)$ & $b^{\prime} \leftarrow \Phi \mathcal{A}\left(1^{\lambda}, \mathbf{p k}, \mathbf{c}, a\right) ; \operatorname{Return}\left(b=b^{\prime}\right)$ \\
\hline
\end{tabular}

Figure 6: Games defining one-wayness of function family $F$ relative to target generator $X$ and PRIV1-security of deterministic public-key encryption scheme DPKE relative to target generator $\mathrm{X}$.

We will now prove that $\operatorname{Adv}_{\mathrm{Obf}, \mathcal{S}^{\mathrm{s}}, \mathcal{O}^{\mathrm{s}}}^{\mathrm{io}}(\lambda)$ is negligible by showing that $\mathrm{S}^{\mathrm{s}} \in \boldsymbol{S}_{\text {diff }}(d)$. Since $n=\operatorname{S} \cdot \mathrm{vl}(\lambda)$ is a polynomial, it will follow that the above sum is negligible. Given a PT adversary $\mathcal{D}^{s}$ we construct a PT adversary $\mathcal{D}^{\mathrm{m}}$ such that $\operatorname{Adv}_{\mathrm{S}^{\mathrm{m}}, \mathcal{D}^{\mathrm{m}}}^{\mathrm{m} \text {-diff }}(\lambda) \geq \operatorname{Adv}_{\mathrm{S}^{\mathrm{s}}, \mathcal{D}^{\mathrm{s}}}^{\text {diff }}(\lambda)$.

$$
\begin{aligned}
& \text { Adversary } \mathcal{D}^{\mathrm{m}}\left(1^{\lambda}, \mathbf{C}_{0}, \mathbf{C}_{1}, a u x^{\mathrm{m}}\right) \\
& n \leftarrow \mathrm{S} \cdot \mathrm{vl}(\lambda) ; \ell \leftarrow \$\{1, \ldots, n\} \\
& \text { For } i=1, \ldots, \ell-1 \text { do } \overline{\mathbf{C}}[i] \leftarrow \$ \operatorname{Obf}\left(1^{\lambda}, \mathbf{C}_{0}[i]\right) \\
& \text { For } i=\ell+1, \ldots, n \text { do } \overline{\mathbf{C}}[i] \leftarrow \$ \operatorname{Obf}\left(1^{\lambda}, \mathbf{C}_{1}[i]\right) \\
& e \leftarrow \$\{0,1\} ; \overline{\mathbf{C}}_{l} \leftarrow \overline{\mathbf{C}}[1], \ldots, \overline{\mathbf{C}}[\ell-1] ; \overline{\mathbf{C}}_{r} \leftarrow \overline{\mathbf{C}}[\ell+1], \ldots, \overline{\mathbf{C}}[n] \\
& \text { aux }^{\mathrm{s}} \leftarrow\left(\overline{\mathbf{C}}_{l}, \overline{\mathbf{C}}_{r}, e, a u x^{\mathrm{m}}\right) ; x \leftarrow \$ \mathcal{D}^{\mathrm{s}}\left(\mathbf{C}_{0}[\ell], \mathbf{C}_{1}[\ell], a u x^{\mathrm{s}}\right) ; \text { Return } x
\end{aligned}
$$

Now, $\mathbf{S}^{\mathbf{s}} \in \boldsymbol{S}_{\text {diff }}(d)$ follows from the assumption that $\mathbf{S}^{\mathrm{m}} \in \boldsymbol{S}_{\mathrm{m} \text {-diff }}(d)$, which concludes the proof.

\section{Generic constructions of PO}

Prior constructions have targeted IND $[\mathbf{X}]$ for specific choices of $\mathbf{X}$ in ad hoc ways and used nonstandard assumptions. In this section we provide constructions that are generic. This means they take an arbitrary, given class $\mathbf{X}$ of target generators and return a point-function obfuscator that is $\mathrm{IND}[\mathbf{X}]$-secure.

\subsection{PO from iO}

OWFs. Consider game OWF of Fig. [ associated to a function family $F$, a target generator $\mathbf{X}$ with $\mathbf{X} . \mathrm{tl}=\mathrm{F}$.il, and an adversary $\mathcal{F}$. For $\lambda \in \mathbb{N}$ let $\operatorname{Adv}_{\mathrm{F}, \mathrm{X}, \mathcal{F}}^{\mathrm{owf}}(\lambda)=\operatorname{Pr}\left[\mathrm{OWF}_{\mathrm{F}, \mathrm{X}}^{\mathcal{F}}(\lambda)\right]$. Let $\mathbf{X}$ be a class of target generators with target length F.il. Let $X^{1 \mathrm{ur}}$ be the target generator with $X^{1 \mathrm{ur}} \cdot \mathrm{vl}(\cdot)=1$ and $\mathrm{X}^{1 \mathrm{ur}} . \mathrm{tl}=\mathrm{F}$.il, where the target is sampled from a uniform distribution and the auxiliary information is always empty, meaning $a=\varepsilon$. We say that $\mathrm{F}$ is $\operatorname{OWF}[\mathbf{X}]$-secure if $\operatorname{Adv}_{\mathrm{F}, \mathbf{X}, \mathcal{F}}^{\text {owf }}(\cdot)$ is negligible for all PT adversaries $\mathcal{F}$ and all $\mathbf{X} \in \mathbf{X} \cup\left\{\mathbf{X}^{1 \text { ur }}\right\}$. Relevant classes $\mathbf{X}$ are the same as for PO. The standard notion of a OWF is recovered as $\mathbf{X}=\emptyset$, meaning that $F$ is secure only with respect to $X^{1 \text { ur }}$.

The definition of CD [24] is the special case of ours with vectors of length one. That of FOR [32], like ours, considers evaluations of the function on multiple inputs, but in their case the key for the evaluations is the same and there is no auxiliary input, while in our case the key is independently 


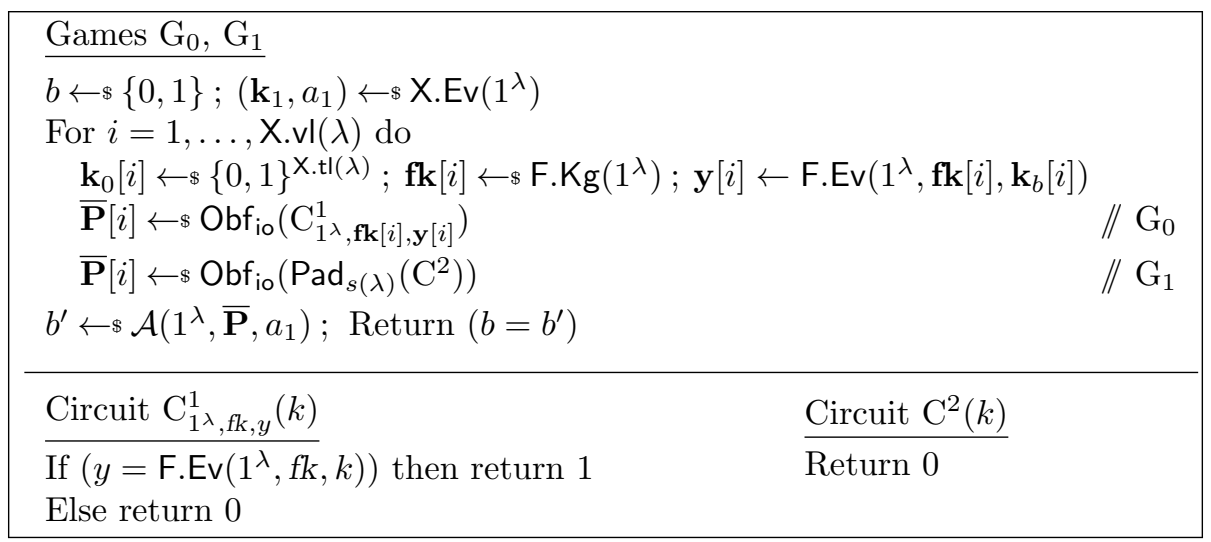

Figure 7: Games for proof of Theorem 5.7.

chosen for each evaluation and auxiliary inputs may be present. We stress that we require only one-wayness; we do not require extractability. The latter is a much stronger assumption [13].

We now show that indistinguishability obfuscation can be used to build a $\operatorname{IND}[\mathbf{X}]$-secure pointfunction obfuscator for an arbitrary target generator class $\mathbf{X}$ from any $\mathrm{OWF}[\mathbf{X}]$-secure function family.

Construction. Let $F$ be a family of functions. Let $\mathrm{Obf}_{\text {io }}$ be an obfuscator. We construct a point-function obfuscator Obf with Obf.tl = F.il as follows:

\begin{tabular}{|c|c|}
\hline $\operatorname{Algorithm~} \operatorname{Obf}\left(1^{\lambda}, \mathbf{I}_{k}\right)$ & Circuit $\mathrm{C}_{1^{\lambda}, f k, y}\left(k^{\prime}\right)$ \\
\hline$f k \leftarrow \$ \mathrm{~F} \cdot \mathrm{Kg}\left(1^{\lambda}\right) ; y \leftarrow \mathrm{F} \cdot \operatorname{Ev}\left(1^{\lambda}, f k, k\right)$ & 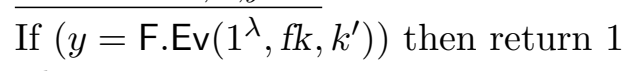 \\
\hline$\overline{\mathrm{P}} \leftarrow \$ \mathrm{Obf}_{\mathrm{io}}\left(\mathrm{C}_{1^{\lambda}, f k, y}\right) ;$ Return $\overline{\mathrm{P}}$ & Else return 0 \\
\hline
\end{tabular}

Theorem 5.1 Let $\mathrm{F}$ be an injective family of functions. Let $\mathbf{X}$ be a class of target generators with target length $\mathrm{F}$.il. Assume that $\mathrm{F}$ is $\mathrm{OWF}[\mathbf{X}]$-secure. Let $\mathrm{Obf}_{\mathrm{io}}$ be an indistinguishability obfuscator. Then $\mathrm{Obf}$ constructed above from $\mathrm{F}$ and $\mathrm{Obf}_{\mathrm{io}}$ is a $\mathrm{IND}[\mathbf{X}]$-secure point-function obfuscator.

Proof of Theorem 5.1: The injectivity of F implies that Obf satisfies the correctness condition of a point-function obfuscator. We now prove security.

Let $\mathrm{X} \in \mathbf{X}$ be a target generator. Let $\mathcal{A}$ be a PT adversary. Consider the games and the associated circuits of Fig. $\mathbb{Z}$, where $s$ is defined as follows. For any $\lambda$ let $s(\lambda)$ be a polynomial upper bound on $\max \left(\left|\mathrm{C}_{1^{\lambda}, f k, y}^{1}\right|\right)$, where the maximum is over all $f k \in\left[\mathrm{F} \cdot \operatorname{Kg}\left(1^{\lambda}\right)\right]$ and $y \in\{0,1\}^{\mathrm{F} . \mathrm{ol}(\lambda)}$. Lines not annotated with comments are common to all games.

Game $\mathrm{G}_{0}$ is equivalent to $\operatorname{IND}_{\text {Obf,X }}^{\mathcal{A}}(\lambda)$. The inputs to adversary $\mathcal{A}$ in game $\mathrm{G}_{1}$ do not depend on the challenge bit $b$, so we have $\operatorname{Pr}\left[\mathrm{G}_{1}\right]=1 / 2$. It follows that

$$
\operatorname{Adv}_{\mathrm{Obf}, \mathrm{X}, \mathcal{A}}^{\mathrm{ind}}(\lambda)=2 \cdot \operatorname{Pr}\left[\mathrm{G}_{0}\right]-1=2 \cdot\left(\operatorname{Pr}\left[\mathrm{G}_{0}\right]-\operatorname{Pr}\left[\mathrm{G}_{1}\right]\right) .
$$

The first equality holds by the definition of IND, and the second equality holds because of $\operatorname{Pr}\left[\mathrm{G}_{1}\right]=$ $1 / 2$. We now show that $\operatorname{Pr}\left[\mathrm{G}_{0}\right]-\operatorname{Pr}\left[\mathrm{G}_{1}\right]$ is negligible, meaning that $\operatorname{Adv}_{\mathrm{Obf}, \mathrm{X}, \mathcal{A}}^{\text {ind }}(\cdot)$ is also negligible. This proves the the theorem.

We construct a multi-circuit sampler $\mathrm{S}$ and a PT iO-adversary $\mathcal{O}$ as follows: 


\begin{tabular}{|c|c|}
\hline Multi-circuit Sampler $S\left(1^{\lambda}\right)$ & Adversary $\mathcal{O}\left(1^{\lambda}, \overline{\mathbf{C}}\right.$, aux $)$ \\
\hline 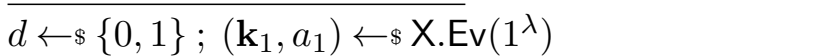 & $\left(d, a_{1}\right) \leftarrow a u x$ \\
\hline For $i=1, \ldots, \mathrm{X} \cdot \mathrm{vl}(\lambda)$ do & $d^{\prime} \leftarrow \$ \mathcal{A}\left(1^{\lambda}, \overline{\mathbf{C}}, a_{1}\right)$ \\
\hline $\mathbf{k}_{0}[i] \leftarrow \$\{0,1\}^{\mathrm{X} \cdot \mathrm{tl}(\lambda)}$ & If $\left(d=d^{\prime}\right)$ then return 1 \\
\hline $\begin{array}{l}\mathbf{f k}[i] \leftarrow \$ \mathrm{~F} . \operatorname{Kg}\left(1^{\lambda}\right) ; \mathbf{y}[i] \leftarrow \mathrm{F} . \operatorname{Ev}\left(1^{\lambda}, \mathbf{f k}[i], \mathbf{k}_{d}[i]\right) \\
\mathbf{C}_{1}[i] \leftarrow \mathrm{C}_{1 \lambda}^{1} \mathbf{f t}[i]\end{array}$ & Else return 0 \\
\hline aux $\leftarrow\left(d, a_{1}\right) ;$ Return $\left(\mathbf{C}_{0}, \mathbf{C}_{1}\right.$, aux $)$ & \\
\hline
\end{tabular}

We have $\operatorname{Pr}\left[G_{0}\right]-\operatorname{Pr}\left[G_{1}\right]=\operatorname{Adv}_{\mathrm{Obf}_{\mathrm{io}}, \mathrm{S}, \mathcal{O}}^{\mathrm{O} \text {-io }}(\lambda)$ by construction. Next, we show that $\mathrm{S} \in \boldsymbol{S}_{\mathrm{m} \text {-diff }}(1)$. According to Proposition [2.] (the result of BCP [16]), any indistinguishability obfuscator is also an $\boldsymbol{S}_{\text {diff }}(1)$-secure obfuscator. And according to Lemma 1.$]$, any $\boldsymbol{S}_{\text {diff }}(1)$-secure obfuscator is an $\boldsymbol{S}_{\mathrm{m} \text {-diff }}(1)$-secure obfuscator. It follows that $\operatorname{Adv}_{\mathrm{Obf}_{\mathrm{i}}, \mathrm{S}, \mathcal{O}}^{\mathrm{m}-\mathrm{O}}(\cdot)$ is negligible by the iO-security of Obf $\mathrm{io}_{\mathrm{io}}$.

Let $X^{\text {ur }}$ be the target generator with $X^{\text {ur }} . v l=X . v l$ and $X^{u r} . t l=F . i l$, where the targets are sampled independently, from a uniform distribution and auxiliary information is always $a=\varepsilon$. Given any PT difference adversary $\mathcal{D}$ against multi-circuit sampler $\mathrm{S}$, we build $\mathrm{PT}$ adversaries $\mathcal{F}_{0}$ and $\mathcal{F}_{1}$ against the OWF-security of $F$ relative to target generators $X^{u r}$ and $X$, respectively. The constructions are as follows:

$$
\begin{array}{l|l}
\text { Adversary } \mathcal{F}_{0}\left(1^{\lambda}, \mathbf{f k}, \mathbf{y}, a\right) & \text { Adversary } \mathcal{F}_{1}\left(1^{\lambda}, \mathbf{f k}, \mathbf{y}, a\right) \\
\hline d \leftarrow 0 ;\left(\mathbf{k}_{1}, a_{1}\right) \leftarrow \$ \mathrm{X} . \operatorname{Ev}\left(1^{\lambda}\right) & d \leftarrow 1 \\
\begin{array}{ll}
\text { For } i=1, \ldots,|\mathbf{y}| \text { do } \\
\quad \mathbf{C}_{1}[i] \leftarrow \mathrm{C}_{1 \lambda}^{1}, \mathbf{f k}[i], \mathbf{y}[i]
\end{array} & \text { For } i=1, \ldots,|\mathbf{y}| \text { do } \\
\quad \mathbf{C}_{0}[i] \leftarrow \operatorname{Pad}_{s(\lambda)}\left(\mathrm{C}^{2}\right) & \mathbf{C}_{0}[i] \leftarrow \mathrm{C}_{1}^{1}, \mathbf{f k}[i], \mathbf{y}[i] \\
\text { aux } \leftarrow\left(d, a_{1}\right) ; x \leftarrow \$ \mathcal{D}\left(\mathbf{C}_{1}, \mathbf{C}_{0}, \text { aux }\right) & \text { aux } \leftarrow(d, a) ; x \leftarrow \$ \mathcal{D}\left(\mathbf{C}_{1}, \mathbf{C}_{0}, \text { aux }\right) \\
\text { Return } x & \text { Return } x
\end{array}
$$

Let $d$ denote the value sampled by multi-circuit sampler $S$ in game $\operatorname{MDIFF}_{\mathrm{S}}^{\mathcal{D}}(\lambda)$. Then we have

$$
\begin{aligned}
& \operatorname{Pr}\left[\operatorname{MDIFF}_{\mathrm{S}}^{\mathcal{D}}(\lambda) \mid d=0\right]=\operatorname{Pr}\left[\operatorname{OWF}_{\mathrm{F}, \mathbf{X}^{\text {ur }}}^{\mathcal{F}_{0}}(\lambda)\right], \\
& \operatorname{Pr}\left[\operatorname{MDIFF}_{\mathrm{S}}^{\mathcal{D}}(\lambda) \mid d=1\right]=\operatorname{Pr}\left[\operatorname{OWF}_{\mathrm{F}, \mathbf{X}}^{\mathcal{F}_{1}}(\lambda)\right] .
\end{aligned}
$$

and $\operatorname{Adv}_{\mathrm{S}, \mathcal{D}}^{\mathrm{m} \text {-diff }}(\lambda)=\frac{1}{2}\left(\operatorname{Adv}_{\mathrm{F}, \mathbf{X}^{\mathrm{ur}}, \mathcal{F}_{0}}^{\mathrm{owf}}(\lambda)+\operatorname{Adv}_{\mathrm{F}, \mathrm{X}, \mathcal{F}_{1}}^{\mathrm{owf}}(\lambda)\right)$. Note that $\mathrm{OWF}[\mathbf{X}]$-security of $\mathrm{F}$ requires that $\operatorname{Adv}_{\mathrm{F}, \mathrm{X}^{1 \mathrm{ur}}, \mathcal{F}}^{\text {oow }}(\lambda)$ is negligible for all PT adversaries $\mathcal{F}$. One can use the latter with a standard hybrid argument to further prove that $\operatorname{Adv}_{\mathrm{F}, \mathrm{X}^{\mathrm{ur}}, \mathcal{F}_{0}}^{\text {owf }}(\lambda)$ is also negligible for all PT adversaries $\mathcal{F}_{0}$. It follows that the multi-circuit sampler $S$ is difference-secure. The injectivity of $F$ also implies that $\mathrm{S}$ produces 1-differing circuits. Therefore, $\mathrm{S} \in \boldsymbol{S}_{\mathrm{m} \text {-diff }}(1)$.

\subsection{PO from DPKE}

Our next generic construction is based on deterministic public-key encryption [3]. As before we aim to provide point-function obfuscation secure for any given class of target generators. We are able to do this assuming the existence of a deterministic public-key encryption scheme that is secure relative to the same class viewed as a class of message generators. We can then exploit known constructions of deterministic public-key encryption to get a slew of point-function obfuscators based on standard assumptions. We begin with a parameterized definition of security for deterministic public-key encryption. 
DPKE. A deterministic public-key encryption scheme DPKE [3] specifies the following. PT key generation algorithm DPKE.Kg takes $1^{\lambda}$ to return a public encryption key pk and a secret decryption key sk. Deterministic PT encryption algorithm DPKE.Enc takes $1^{\lambda}$, pk and a plaintext message $k \in\{0,1\}^{\text {DPKE.ml }(\lambda)}$ to return a ciphertext $c$, where DPKE.ml: $\mathbb{N} \rightarrow \mathbb{N}$ is the message length function associated to DPKE. Deterministic decryption algorithm DPKE.Dec takes $1^{\lambda}$, sk, $c$ to return plaintext message $k$. We do not require the decryption algorithm to be $\mathrm{PT}$ but we do require decryption correctness, namely that for all $\lambda \in \mathbb{N}$, all $(p k, s k) \in\left[\mathrm{DPKE} . \operatorname{Kg}\left(1^{\lambda}\right)\right]$ and all $k \in\{0,1\}^{\mathrm{DPKE} \cdot \mathrm{ml}(\lambda)}$ we have DPKE.Dec $\left(1^{\lambda}, \mathrm{sk}\right.$, DPKE.Enc $\left.\left(1^{\lambda}, p k, k\right)\right)=k$.

Now consider game PRIV1 of Fig. 6 associated to a deterministic public-key encryption scheme DPKE, a target generator $\mathrm{X}$ satisfying $\mathrm{X} . \mathrm{tl}=\mathrm{DPKE} . \mathrm{ml}$, and an adversary $\mathcal{A}$. For $\lambda \in \mathbb{N}$ let $\operatorname{Adv}_{\mathrm{DPKE}, \mathrm{X}, \mathcal{A}}^{\text {priv }}(\lambda)=2 \operatorname{Pr}\left[\operatorname{PRIV} 1_{\mathrm{DPKE}, \mathrm{X}}^{\mathcal{A}}(\lambda)\right]-1$. If $\mathbf{X}$ is a class of target generators then we say that DPKE is PRIV1 $[\mathbf{X}]$-secure if $\operatorname{Adv}_{\mathrm{DPKE}, \mathbf{X}, \mathcal{A}}^{\text {priv1 }}(\cdot)$ is negligible for all PT adversaries $\mathcal{A}$ and all $\mathbf{X} \in \mathbf{X}$.

This definition reflects what BBO [3] call the multi-user setting where there are many, independent public keys. However, in our case, only a single message is encrypted under each key. The single-key version of this is called PRIV1 in the literature, so we retained the name in moving to the multi-user setting. The definition is in the indistinguishability style of [4, [1.5] rather than the semantic security style of [3]. These definitions however did not allow auxiliary inputs. We are allowing those following BS [17]. Finally, while prior definitions require unpredictability of the message distribution, ours is simply parameterized by the latter. Prior definitions are captured as special cases, meaning they can be recovered as PRIV1[X] for some choice of $\mathbf{X}$.

CONSTRUCTION. Let DPKE be a deterministic public-key encryption scheme. We construct an obfuscator Obf with Obf.tI = DPKE.ml as follows:

$$
\begin{array}{l|l}
\text { Algorithm } \operatorname{Obf}\left(1^{\lambda}, \mathbf{I}_{k}\right) & \text { Circuit } \mathrm{C}_{1^{\lambda}, p k, c}(k) \\
\hline(p k, s k) \leftarrow \$ \operatorname{DPKE} . \operatorname{Kg}\left(1^{\lambda}\right) & \text { If }\left(\operatorname{DPKE} \cdot \operatorname{Enc}\left(1^{\lambda}, p k, k\right)=c\right) \\
c \leftarrow \text { DPKE.Enc }\left(1^{\lambda}, p k, k\right) ; \text { Return } \mathrm{C}_{1^{\lambda}, p k, c} & \text { Then return } 1 \text { else return 0 }
\end{array}
$$

The construction is simple. To obfuscate $\mathbf{I}_{k}$ we pick a new key pair for the deterministic public-key encryption scheme and return a circuit that embeds the public key pk as well as the encryption $c$ of the target point $k$. The circuit, given a candidate target point $k^{\prime}$, re-encrypts it under the embedded public key $p k$ and checks that the ciphertext so obtained matches the embedded ciphertext $c$. Note that the determinism of DPKE.Enc is used crucially to ensure that the circuit is deterministic. For randomized encryption, one cannot check that a message corresponds to a ciphertext by reencryption. The secret key sk is discarded and not used in the construction, but its existence will guarantee correctness of the point-function obfuscator.

RESULT. We show that this is a generic construction. Namely, a point-function obfuscator for a given class $\mathbf{X}$ of target generators can be obtained if we have a deterministic public-key encryption scheme secure for the same class.

Theorem 5.2 Let DPKE be a deterministic public-key encryption scheme and $\mathbf{X}$ a class of target generators such that $\mathbf{X} . \mathrm{tl}=\mathrm{DPKE} . \mathrm{ml}$ for all $\mathbf{X} \in \mathbf{X}$. Assume DPKE is PRIV1[X]-secure. Let Obf be as defined above. Then $\mathrm{Obf}$ is a $\mathrm{IND}[\mathbf{X}]$-secure point-function obfuscator.

Proof of Theorem 5.2]: The correctness of Obf follows from the decryption correctness of DPKE, and it does not require the decryption algorithm DPKE.Dec to be PT. We now prove that Obf is $\operatorname{IND}[\mathbf{X}]$-secure. 
Let $\mathrm{X} \in \mathbf{X}$ be a target generator with $\mathbf{X} . t \mathrm{tl}=$ DPKE.ml. Let $\mathcal{A}$ be PT adversary against the IND security of Obf relative to $X$. We construct a PT adversary $\mathcal{B}$ against the PRIV1 security of DPKE relative to $\mathrm{X}$ as follows:

$$
\begin{array}{l|l}
\text { Adversary } \mathcal{B}\left(1^{\lambda}, \mathbf{p k}, \mathbf{c}, a\right) & \text { Circuit } \mathrm{C}_{1^{\lambda}, p k, c}(k) \\
\hline \text { For } i=1, \ldots,|\mathbf{c}| \text { do } \overline{\mathbf{P}}[i] \leftarrow \mathrm{C}_{1^{\lambda}, \mathbf{p k}[i], \mathbf{c}[i]} & \text { If (DPKE.Enc } \left.\left(1^{\lambda}, p k, k\right)=c\right) \\
b^{\prime} \leftarrow \$ \mathcal{A}\left(1^{\lambda}, \overline{\mathbf{P}}, a\right) ; \text { Return } b^{\prime} & \text { Then return 1 else return 0 }
\end{array}
$$

We have $\operatorname{Adv}_{\mathrm{DPKE}, \mathbf{X}, \mathcal{B}}^{\text {priv1 }}(\lambda)=\operatorname{Adv}_{\mathrm{Obf}, \mathrm{X}, \mathcal{A}}^{\text {ind }}(\lambda)$ by construction. Hence, for any $\mathbf{X} \in \mathbf{X}$ the IND-security of Obf relative to $X$ follows from the assumed PRIV1-security of DPKE relative to $X$.

In applying Theorem 5.2 to get point function obfuscators, the first case of interest is $\mathbf{X}=\mathbf{X}^{\sup } \cap$ $\mathbf{X}^{\varepsilon} \cap \mathbf{X}^{1}$. In this case, PRIV1[X]-secure deterministic public-key encryption is a standard form of the latter for which many constructions are known. The central construction, due to BFO [15], is from lossy trapdoor functions (LTDFs). But the latter can be built from a wide variety of standard assumptions [47, 31, 52, 37, 55]. Thus we get $\operatorname{IND}\left[\mathbf{X}^{\sup } \cap \mathbf{X}^{\varepsilon} \cap \mathbf{X}^{1}\right]$-secure point-function obfuscators under the same assumptions. The second case of interest is $\mathbf{X}=\mathbf{X}^{\text {seup }} \cap \mathbf{X}^{1}$. Unlike in the first case, there is now auxiliary information, but it leaves the targets sub-exponentially unpredictable. Constructions of PRIV1[X]-secure deterministic public-key encryption are known under standard assumptions including DLIN, Subgroup Indistinguishability and LWE [177, 54, 52]. Accordingly we get IND $\left[\mathbf{X}^{\text {seup }} \cap \mathbf{X}^{1}\right]$-secure point-function obfuscators under the same assumptions. BH [5] obtain PRIV-secure DPKE from UCE[S $\left.\mathbf{S}^{\text {sup }}\right]$, which via Theorem 5.2 yields $\operatorname{IND}\left[\mathbf{X}^{\sup } \cap \mathbf{X}^{\varepsilon} \cap \mathbf{X}^{1}\right]$ under $\mathrm{UCE}\left[\mathbf{S}^{\text {sup }}\right]$.

Theorem 5.2 also yields negative results. Assume $\mathrm{iO}$ exists. Then we know that there do not exist point function obfuscators that are $\operatorname{IND}\left[\mathbf{X}^{\text {cup }}\right]$-secure [201, 24]]. Theorem 52 then implies that there also do not exist deterministic public-key encryption schemes that are PRIV1[ $\left.\mathbf{X}^{\text {cup }}\right]$-secure.

CIH function families as per GOR [36] do not seem to have a unique associated security notion. Rather the authors discuss a few choices. Our parameterized PRIV definitions above apply to function families as well and can be viewed as providing more security notions for CIH function families. These function families can also be used in our PO construction above as long as they are injective.

\subsection{PO from UCE}

Our next generic construction is based on UCE, a class of assumptions on function families from [6]. We use the multi-key version of the UCE assumption, denoted mUCE. As before we aim to provide point-function obfuscation secure for any given class of target generators. We are able to do this with mUCE by associating to the class of target generators a class of sources. The existence of an mUCE-secure function family relative to the latter suffices to construct a point-function obfuscator secure relative to the former.

Construction. Let $\mathrm{H}$ be a family of functions. Associate to it a point-function obfuscator Obf defined as follows. Let Obf.tl $=$ H.il, and

$$
\begin{array}{l|l}
\text { Algorithm } \operatorname{Obf}\left(1^{\lambda}, \mathbf{I}_{k}\right) & \operatorname{Circuit~} \mathrm{C}_{1^{\lambda}, h k, y}\left(k^{\prime}\right) \\
\hline h k \leftarrow \$ \mathrm{H} . \mathrm{Kg}\left(1^{\lambda}\right) ; y \leftarrow \mathrm{H} . \operatorname{Ev}\left(1^{\lambda}, h k, k\right) & \begin{array}{l}
y^{\prime} \leftarrow \mathrm{H} \cdot \operatorname{Ev}\left(1^{\lambda}, h k, k^{\prime}\right) \\
\text { Return } \mathrm{C}_{1^{\lambda}, h k, y}
\end{array}
\end{array}
$$

The construction is simple and natural. The point-function obfuscation of $\mathbf{I}_{k}$ is a circuit that embeds the hash $y$ of target $k$ under a freshly-chosen key $h k$ also embedded in the circuit, and, given a candidate target $k^{\prime}$, checks whether its hash under hk equals the embedded hash value. 
Source ClASsES. To state the result, we need a few definitions. Associate to a target generator $\mathrm{X}$ a source $\mathcal{S}^{\mathrm{X}}$ defined as follows:

$$
\begin{aligned}
& \text { Source } \mathcal{S}^{\mathrm{X}}\left(1^{\lambda}\right) \\
& d \leftarrow \$\{0,1\} ;\left(\mathbf{k}_{1}, a_{1}\right) \leftarrow \$ \mathrm{X} \cdot \operatorname{Ev}\left(1^{\lambda}\right) \\
& \text { For } i=1, \ldots, \mathrm{X} \cdot \mathrm{vl}(\lambda) \text { do } \mathbf{k}_{0}[i] \leftarrow \$\{0,1\}^{\mathrm{X} . \mathrm{tl}(\lambda)} \\
& \text { For } i=1, \ldots, \mathrm{X} \cdot \mathrm{vl}(\lambda) \text { do } \mathbf{y}[i] \leftarrow \$ \operatorname{HASH}\left(i, \mathbf{k}_{d}[i]\right) \\
& L \leftarrow\left(\left(d, a_{1}\right), \mathbf{y}\right) ; \text { Return } L
\end{aligned}
$$

where HASH is a hash oracle that is provided to $\mathcal{S}^{\mathrm{X}}$ in mUCE security game. The number of keys for this source is $\mathcal{S}^{\mathbf{X}} . n k=X . v l$, the number of points in the target vector. Now let $\mathbf{X}$ be a class of target generators and let $\mathbf{S}^{\mathbf{X}}=\left\{\mathcal{S}^{\mathbf{X}}: \mathbf{X} \in \mathbf{X}\right\}$ be the corresponding class of sources. We will show that the construction above is $\operatorname{IND}[\mathbf{X}]$-secure assuming $\mathbf{H}$ is $\operatorname{mUCE}\left[\mathbf{S}^{\mathbf{X}}\right]$-secure. To appreciate what this provides we now discuss the assumption further.

Assumptions in the UCE framework are very sensitive to the class of sources for which security is assumed. Accordingly one tries to restrict sources in different ways. In this regard $\mathbf{S}^{\mathbf{X}}=\left\{\mathcal{S}^{\mathbf{X}}\right.$ : $\mathbf{X} \in \mathbf{X}\}$ has some good attributes as we now discuss, referring to definitions of classes of mUCE sources recalled in Section 2 .

The first attribute is that the sources in $\mathbf{S}^{\mathbf{X}}$ are what BHK [6] call "split," so that $\mathbf{S}^{\mathbf{X}} \subseteq$ $\mathbf{S}^{\text {splt }}$. "Split" means that the leakage is a function of the oracle queries and answers separately, but not both together. (Above, $\left(d, a_{1}\right)$ depends only on the oracle queries, and $\mathbf{y}$ depends only on the answers.) The second attribute is that the sources make only one query per key. (In particular when there is only one target point, the source makes only one query overall.) That is, $\mathbf{S}^{\mathbf{X}} \subseteq \mathbf{S}^{n, 1}$ if $\mathcal{S} . n k(\cdot) \leq n(\cdot)$ for all $\mathcal{S} \in \mathbf{S}^{\mathbf{X}}$. The third attribute is that the source class inherits the unpredictability properties of the target generator class. Thus if $\mathbf{X} \subseteq \mathbf{X}^{\text {cup }}$ then $\mathbf{S}^{\mathbf{X}} \subseteq \mathbf{S}^{\text {cup }}$ consists of computationally unpredictable sources; if $\mathbf{X} \subseteq \mathbf{X}^{\text {sup }}$ then $\mathbf{S}^{\mathbf{X}} \subseteq \mathbf{S}^{\text {sup }}$ consists of statistically unpredictable sources; and if $\mathbf{X} \subseteq \mathbf{X}^{\text {seup }}$ then $\mathbf{S}^{\mathbf{X}} \subseteq \mathbf{S}^{\text {seup }}$ consists of sources that are sub-exponentially unpredictable.

We warn that $\mathrm{mUCE}\left[\mathbf{S}^{\mathbf{X}}\right]$-security is not achievable for all choices of $\mathbf{X}$. The value of our result is that it is entirely general, reducing IND security for a given $\mathbf{X}$ to a question of mUCE security for a related class of sources, and we can then investigate the latter separately. In this way we get many new constructions.

RESULT. The following theorem shows that our construction above provides secure point-function obfuscation in a very general and modular way, namely the point-function obfuscator is secure relative to a class of target generators if $\mathrm{H}$ is mUCE-secure relative to the corresponding class of sources. After stating and proving this general result we will look at some special cases of interest.

Theorem 5.3 Let $\mathrm{H}$ be an injective family of functions. Let $\mathbf{X}$ be a class of target generators such that $\mathbf{X} . \mathrm{tl}=\mathrm{H}$.il for all $\mathbf{X} \in \mathbf{X}$. Assume $\mathrm{H}$ is $\mathrm{mUCE}\left[\mathbf{S}^{\mathbf{X}}\right]$-secure. Let $\mathrm{Obf}$ be as defined above. Then Obf is a $\mathrm{IND}[\mathbf{X}]$-secure point-function obfuscator.

Function family $\mathrm{H}$ is assumed to be injective in order to meet the perfect correctness condition of a point-function obfuscator, and it is not important for security. In Appendix $⿴ 囗$ 业 we show that non-injective $\mathrm{mUCE}$ is sufficient to construct a point-function obfuscator that satisfies a relaxed correctness condition and achieves the same security as above.

Proof of Theorem [5.3: Correctness of the obfuscator follows from the assumed injectivity of $\mathrm{H}$, meaning that the output of $\operatorname{Obf}\left(1^{\lambda}, \mathbf{I}_{k}\right)$ is always a point circuit with target $k$. We now prove that Obf is IND $[\mathbf{X}]$-secure. 
Let $\mathrm{X} \in \mathrm{X}$ be any target generator with $\mathrm{X} . \mathrm{tl}=\mathrm{H}$.il. Let $\mathcal{S}^{\mathrm{X}}$ be the corresponding source as defined above. Let $\mathcal{A}$ be a PT adversary against the IND-security of Obf relative to X. We define a PT distinguisher $\mathcal{D}$ as follows:

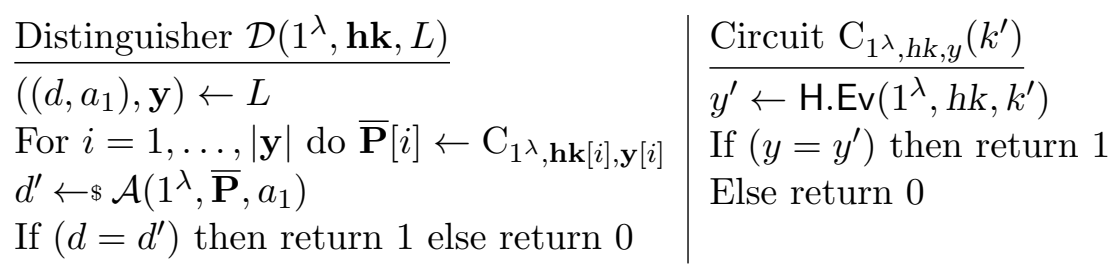

Let $b$ denote the challenge bit in game $\operatorname{mUCE}_{\mathrm{H}}^{\mathcal{S}^{\mathrm{x}}, \mathcal{D}}(\lambda)$, and let $b^{\prime}$ denote the bit returned by $\mathcal{D}$ in the same game. We claim that

$$
\operatorname{Pr}\left[b^{\prime}=1 \mid b=1\right]=\operatorname{Pr}\left[\operatorname{IND}_{\mathrm{Obf}, \mathrm{X}}^{\mathcal{A}}(\lambda)\right] \quad \text { and } \quad \operatorname{Pr}\left[b^{\prime}=1 \mid b=0\right]=\frac{1}{2} .
$$

The first equation holds by construction. The second equation is true because $\mathcal{D}$ runs $\mathcal{A}$ with inputs that are independent of the challenge bit $d$. Namely, for $b=0$ the entries in $\mathbf{y}$ are uniform and independent, since the source $\mathcal{S}$ makes only one query per key index. We have $\operatorname{Adv}_{\mathrm{H}, \mathcal{S}^{\mathrm{x}}, \mathcal{D}}^{\mathrm{m}}(\lambda)=$ $\operatorname{Adv}_{\mathrm{Obf}, \mathrm{X}, \mathcal{A}}^{\mathrm{ind}}(\lambda) / 2$. Therefore, for any $\mathbf{X} \in \mathbf{X}$ the IND security of Obf relative to $\mathbf{X}$ follows from the assumed mUCE $\left[\left\{\mathcal{S}^{\mathrm{X}}\right\}\right]$-security of $\mathrm{H}$.

NeGATIVE RESUlts FOR MULTI-Key UCE. Let $n: \mathbb{N} \rightarrow \mathbb{N}$ be a polynomial such that $n(\cdot) \in$ $\Omega\left((\cdot)^{\epsilon}\right)$. We claim that Theorem 5.3 allows us to conclude that $\operatorname{mUCE}\left[\mathbf{S}^{\text {cup }} \cap \mathbf{S}^{\text {splt }} \cap \mathbf{S}^{n, 1}\right]$-secure injective function families do not exist under certain assumptions. This is a simple corollary of the prior work that shows that MB-AIPO can not co-exist with iO [2.5, 20]. We now explain our claim in more details.

Theorem 5.3 shows that the existence of $\operatorname{mUCE}\left[\mathbf{S}^{\text {cup }} \cap \mathbf{S}^{\text {splt }} \cap \mathbf{S}^{n, 1}\right]$-secure injective function families implies IND $\left[\mathbf{X}^{\text {cup }} \cap \mathbf{X}^{n}\right]$-secure point-function obfuscation. Note that the latter is a composable AIPO as per CD [25]. CD [25] show that composable AIPO can be used to construct MB-AIPO, which is an obfuscation that is secure for functions that map a target point to a multi-bit output (as opposed to an output in $\{0,1\}$ ). Finally, BM1 [20] show that MB-AIPO cannot co-exist with $\mathrm{iO}$, assuming one-way functions. Together these results imply the following:

Corollary 5.4 Let $\mathrm{H}$ be an injective function family. Let $n: \mathbb{N} \rightarrow \mathbb{N}$ be a polynomial such that $n(\cdot) \in \Omega\left((\cdot)^{\epsilon}\right)$ for some constant $\epsilon>0$. Assume the existence of one-way functions and indistinguishability obfuscation. Then $\mathrm{H}$ is not $\mathrm{mUCE}\left[\mathbf{S}^{\text {cup }} \cap \mathbf{S}^{\text {splt }} \cap \mathbf{S}^{n, 1}\right]$-secure.

In a concurrent and independent work, BM3 [22] discuss a similar impossibility result for mUCE[ $\left.\mathbf{S}^{\mathbf{s}^{-c u p}} \cap \mathbf{S}^{n, 1}\right]$-security. Here $\mathbf{S}^{\mathbf{s}^{-c u p}}$ is a class of UCE sources introduced in (BM2) [21] who also show that $\mathbf{S}^{\text {cup }} \cap \mathbf{S}^{\text {splt }} \subsetneq \mathbf{S}^{\text {s-cup }}$. We note that impossibility of mUCE[ $\left[\mathbf{S}^{\text {cup }} \cap \mathbf{S}^{\text {splt }} \cap \mathbf{S}^{n, 1}\right]$-secure function families is a stronger result because it concerns a smaller class of sources.

No other impossibility results are known for mUCE exclusively, but any negative results for

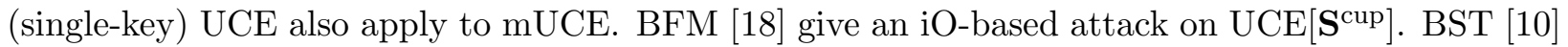
give a strictly stronger impossibility result showing that $\mathrm{UCE}\left[\mathbf{S}^{\text {cup }} \cap \mathbf{S}^{\text {splt }}\right]$-secure function families do not exist assuming the existence of OWFs and iO. The latter implies that mUCE[ $\left[\mathbf{S}^{\text {cup }} \cap \mathbf{S}^{\text {splt }} \cap \mathbf{S}^{1, p}\right]$ secure function families do not exist for a polynomial $p(\cdot) \in \Omega\left((\cdot)^{\epsilon}\right)$, but we currently do not know whether this notion is comparable to $\operatorname{mUCE}\left[\mathbf{S}^{\text {cup }} \cap \mathbf{S}^{\text {splt }} \cap \mathbf{S}^{n, 1}\right]$.

Related WORK. One special case of Theorem [5.3 is when $\mathbf{X}=\mathbf{X}^{\text {cup }} \cap \mathbf{X}^{1}$, so that $\operatorname{IND}[\mathbf{X}]$ is AIPO. The theorem and the remarks preceding it imply that we get this assuming mUCE $\left[\mathbf{S}^{\text {cup }} \cap \mathbf{S}^{\text {splt }} \cap \mathbf{S}^{1,1}\right]$ - 


\begin{tabular}{|c|c|c|}
\hline Game $\operatorname{SIND}_{\mathrm{Obf}, \mathrm{X}}^{\mathcal{A}, \mathcal{D}}(\lambda)$ & Game $\operatorname{CSS}_{\mathrm{Obf}, \mathrm{X}}^{\mathcal{A}}(\lambda)$ & 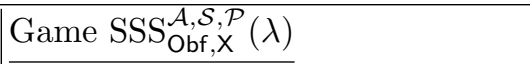 \\
\hline$b \leftarrow \$\{0,1\}$ & $b \leftarrow^{\$}\{0,1\}$ & $b \leftarrow \$\{0,1\}$ \\
\hline$\left(\mathbf{k}_{1}, a_{1}\right) \leftarrow{ }_{8} \mathrm{X} \cdot \operatorname{Ev}\left(1^{\lambda}\right)$ & $\left(\mathbf{k}_{1}, a_{1}\right) \leftarrow \$ \mathrm{X} \cdot \operatorname{Ev}\left(1^{\lambda}\right)$ & $(\mathbf{k}, a) \leftarrow \$ \mathrm{X} \cdot \operatorname{Ev}\left(1^{\lambda}\right)$ \\
\hline For $i=1, \ldots, \mathrm{X} \cdot \mathrm{vl}(\lambda)$ do & For $i=1, \ldots, \mathrm{X} \cdot \mathrm{vl}(\lambda)$ do & $\overline{\mathbf{P}} \leftarrow{ }^{\prime} \operatorname{Obf}\left(1^{\lambda}, \mathbf{I}_{\mathbf{k}}\right)$ \\
\hline $\mathbf{k}_{0}[i] \leftarrow \$\{0,1\}^{\mathrm{X} \cdot \mathrm{t} \mid(\lambda)}$ & $\mathbf{k}_{0}[i] \leftarrow s\{0,1\}^{\mathrm{X} \cdot \mathrm{tl}(\lambda)}$ & $p \leftarrow{ }^{\infty} \mathcal{P}\left(1^{\lambda}, \mathbf{k}, a\right)$ \\
\hline$\overline{\mathbf{P}} \leftarrow \$ \operatorname{Obf}\left(1^{\lambda}, \mathbf{I}_{\mathbf{k}_{b}}\right)$ & $\overline{\mathbf{P}} \leftarrow{ }^{\infty} \operatorname{Obf}\left(1^{\lambda}, \mathbf{I}_{\mathbf{k}_{b}}\right)$ & If $(b=1)$ then $p^{\prime} \leftarrow \Phi \mathcal{A}\left(1^{\lambda}, \overline{\mathbf{P}}, a\right)$ \\
\hline$d \leftarrow \$ \mathcal{A}\left(1^{\lambda}, \overline{\mathbf{P}}, a_{1}\right)$ & $t \leftarrow \Phi \mathcal{A}_{1}\left(1^{\lambda}, \mathbf{k}_{1}, a_{1}\right)$ & Else $p^{\prime} \leftarrow{ }_{s} \mathcal{S}^{\mathbf{I}_{\mathbf{k}}}\left(1^{\lambda}, a\right)$ \\
\hline$b^{\prime} \leftarrow{ }_{\$} \mathcal{D}\left(1^{\lambda}, \mathbf{k}_{1}, a_{1}, d\right)$ & $t^{\prime} \leftarrow \$ \mathcal{A}_{2}\left(1^{\lambda}, \overline{\mathbf{P}}, a_{1}\right)$ & If $\left(p=p^{\prime}\right)$ then $b^{\prime} \leftarrow 1$ else $b^{\prime} \leftarrow 0$ \\
\hline Return $\left(b=b^{\prime}\right)$ & $\begin{array}{l}\text { If }\left(t=t^{\prime}\right) \text { then } b^{\prime} \leftarrow 1 \text { else } b^{\prime} \leftarrow 0 \\
\text { Return }\left(b=b^{\prime}\right)\end{array}$ & Return $\left(b=b^{\prime}\right)$ \\
\hline
\end{tabular}

Figure 8: Games defining SIND security, CSS security and SSS security of point-function obfuscator Obf relative to target generator $X$.

security. This special case of our result was independently and concurrently obtained in [22]. Note that BM2 [2] $]$ showed that $\mathrm{mUCE}\left[\mathbf{S}^{\text {cup }} \cap \mathbf{S}^{\text {splt }} \cap \mathbf{S}^{1,1}\right]$-security is achievable assuming iO and AIPO. It follows from our result that mUCE[ $\left[\mathbf{S}^{\text {cup }} \cap \mathbf{S}^{\text {splt }} \cap \mathbf{S}^{1,1}\right]$ and AIPO are equivalent, assuming iO.

In Section 5.2 we showed that PRIV1[X]-secure DPKE implies IND $[\mathbf{X}]$-secure point-function obfuscation for any class of target generators $\mathbf{X}$. A natural question to ask is whether PRIV1[X] for any target generator class $\mathbf{X}$ is implied by mUCE for some source class $\mathbf{S}^{\mathbf{X}}$, similar to our result above. This would allow to build PO from UCE in a more modular way, by using the earlier DPKE result as an intermediate step. So let us consider known constructions of DPKE from UCE. BHK [6] show that the EwH construction of a DPKE scheme from [3] is PRIV-secure when the random oracle $(\mathrm{RO})$ is instantiated by a family in a subclass of $\mathbf{S}^{\text {cup }}$, but the latter succumbs to the impossibility result of BFM [18] and in fact under $\mathrm{iO}$ the $\mathrm{RO}$ in $\mathrm{EwH}$ cannot in general be securely instantiated [1.9, 5]. BH [5] obtain PRIV-secure DPKE from UCE[S $\left.{ }^{\text {sup }}\right]$, which via Theorem 5.2 yields IND $\left[\mathbf{X}^{\text {sup }} \cap \mathbf{X}^{\varepsilon} \cap \mathbf{X}^{1}\right]$ under $\mathrm{UCE}\left[\mathbf{S}^{\text {sup }}\right]$.

\section{Alternative security notions for PO}

In Section [3 we defined IND security of point-function obfuscation. It extends security notions that were used for variants of AIPO in [14, 42, 20, 21, 22]. The main difference is that IND is parameterized with a class of target generators, allowing us to unify the treatment of AIPO from the literature.

In this section we provide several alternative security notions for point-function obfuscation, and show relations between them and IND. Specifically, we extend the distributional indistinguishability notion introduced by Canetti [2:3] as well as the notions of average-case [2.3, 27, 2.9, 26, 6] and worst-case [41, 51, 35, 25, П1, 14, 42] simulation-based security for point-function obfuscation. Similar to IND, our extended notions are parameterized with classes of target generators. We also define a novel security notion, called computational semantic security, by adapting the corresponding definition that was used for DPKE in [4] to the setting of point-function obfuscation and parameterizing it in the same way as above. Finally, we discuss the security achieved by our PO constructions from Section 1 with respect to the new notions.

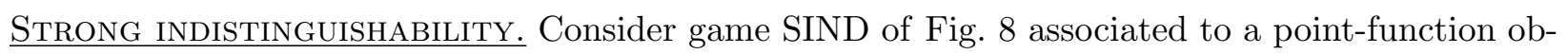
fuscator Obf, a target generator $\mathrm{X}$, an adversary $\mathcal{A}$ and a distinguisher $\mathcal{D}$, such that Obf.tI $=\mathrm{X}$.tl and $\mathcal{A}$ is required to return an output in $\{0,1\}$. For $\lambda \in \mathbb{N}$ let $\operatorname{Adv}_{\mathrm{Obf}, \mathrm{X}, \mathcal{A}, \mathcal{D}}^{\text {sind }}(\lambda)=2 \operatorname{Pr}\left[\operatorname{SiND}_{\mathrm{Obf}, \mathrm{X}}^{\mathcal{A}, \mathcal{D}}(\lambda)\right]-1$. 
Let $\mathbf{X}$ be a class of target generators. We say that $\operatorname{Obf}$ is $\operatorname{SIND}[\mathbf{X}]$-secure if $\operatorname{Adv}_{\mathrm{Obf}, \mathbf{X}, \mathcal{A}, \mathcal{D}}^{\mathrm{sind}}(\cdot)$ is negligible for every $\mathbf{X} \in \mathbf{X}$, every PT $\mathcal{A}$ and every PT $\mathcal{D}$. The difference between our parameterized definitions of IND and SIND is that the latter also runs a distinguisher in the last stage of the game, which makes this definition meaningful even for trivial target generators (as defined in Section B). Our definition of SIND extends the distributional indistinguishability notion used for oracle hashing by Canetti [2:3], parameterizing it with classes of target generators. Another difference is that SIND samples target vectors $\mathbf{k}_{0}, \mathbf{k}_{1}$ from distributions that are potentially different, whereas [2:3] used the same distribution for both. Note that adversary $\mathcal{A}$ cannot be allowed to return an output of an arbitrary length because then it would be able to return $\overline{\mathbf{P}}$, hence making the security trivially unachievable.

Computational SemantiC SECURITY. Consider game CSS of Fig. 8 associated to a point-function obfuscator Obf, a target generator $\mathrm{X}$ and an adversary $\mathcal{A}=\left(\mathcal{A}_{1}, \mathcal{A}_{2}\right)$, such that Obf.tl $=\mathrm{X} . \mathrm{tl}$ and algorithms $\mathcal{A}_{1}, \mathcal{A}_{2}$ are required to return outputs in $\{0,1\}$. For $\lambda \in \mathbb{N}$ let $\operatorname{Adv}_{\mathrm{Obf}, \mathrm{X}, \mathcal{A}}^{\text {css }}(\lambda)=$ $2 \operatorname{Pr}\left[\operatorname{CSS}_{\mathrm{Obf}, \mathrm{X}}^{\mathcal{A}}(\lambda)\right]-1$. Let $\mathbf{X}$ be a class of target generators. We say that Obf is $\operatorname{CSS}[\mathbf{X}]$-secure if $\operatorname{Adv}_{\mathrm{Obf}, \mathrm{X}, \mathcal{A}}^{\mathrm{css}}(\cdot)$ is negligible for every $\mathrm{X} \in \mathbf{X}$ and every $\mathrm{PT} \mathcal{A}$. This is an adaptation of the definition of computational semantic security for DPKE from [4], which we further parameterize with classes of target generators. It asks that adversary $\mathcal{A}$ can not use an obfuscation $\overline{\mathbf{P}}$ of $\mathbf{k}_{1}$ to compute any partial information about the latter, even in the presence of auxiliary information $a_{1}$. This provides a better intuition about the desired security of point-function obfuscation, as opposed to the less intuitive definition of SIND.

Simulation-BASED SECURITY NOTIONS. We consider two different definitions of simulation-based semantic security. Informally, both definitions require that for every $\mathrm{PT}$ adversary $\mathcal{A}$ that receives as input an obfuscation of some point-function $\mathbf{I}_{k}$, there exists a PT simulator with only an oracle access to $\mathbf{I}_{k}$, such that the output distribution of the former is indistinguishable from that of the latter. The two definitions differ in the way how $\mathbf{I}_{k}$ is chosen. One option is to quantify over all possible point-functions that can be produced by a particular target generator. For this purpose, we extend the definitions of worst-case security [41, 51, 35, 25, 11, 14, 42] for point-function obfuscation. We use SIM to denote our new security notion. An alternative approach is to use target generator $\mathrm{X}$ in order to sample point-functions. This follows the definitions of average-case security [2:3, 27, 2.9, 26, 6] for point function obfuscation, and we use SSS to denote our extended security notion.

First, we define the SIM-security of PO. Let $\mathbf{X}$ be a class of target generators. Let Obf be a point-function obfuscator. We say that $\mathrm{Obf}$ is $\operatorname{SIM}[\mathbf{X}]$-secure if for every target generator $\mathbf{X} \in \mathbf{X}$ and every PT adversary $\mathcal{A}$ there exists a PT simulator $\mathcal{S}$ and a negligible function $\mu: \mathbb{N} \rightarrow \mathbb{N}$ such that

$$
\left|\operatorname{Pr}\left[\mathcal{A}\left(1^{\lambda}, \operatorname{Obf}\left(1^{\lambda}, \mathbf{I}_{\mathbf{k}}\right), a\right)=\mathcal{P}(\mathbf{k}, a)\right]-\operatorname{Pr}\left[\mathcal{S}^{\mathbf{I}_{\mathbf{k}}}\left(1^{\lambda}, a\right)=\mathcal{P}(\mathbf{k}, a)\right]\right| \leq \mu(\lambda)
$$

for every $\lambda \in \mathbb{N}$, every $(\mathbf{k}, a) \in\left[\mathrm{X} . \operatorname{Ev}\left(1^{\lambda}\right)\right]$ and every PT predicate algorithm $\mathcal{P}$ that returns an output in $\{0,1\}$. Informally, this security notion requires that for every adversary $\mathcal{A}$ there exists a simulator $\mathcal{S}$ such that if $\mathcal{A}$ can use obfuscations $\mathbf{I}_{\mathbf{k}}$ to compute any property (predicate) $\mathcal{P}$ of $\mathbf{k}$, then $\mathcal{S}$ can do the same using only an oracle access to $\mathbf{I}_{\mathbf{k}}$ (meaning that $\mathcal{S}$ has oracle access to each of $\mathbf{I}_{\mathbf{k}[1]}, \ldots, \mathbf{I}_{\mathbf{k}[n]}$ for $n=|\mathbf{k}|$ ). This is required to hold even when $\mathcal{A}, \mathcal{S}, \mathcal{P}$ receive as input some auxiliary information $a$ about $\mathbf{k}$. It was noted in [2, 51, 35] that predicate $\mathcal{P}$ can be substituted with a constant function, resulting in an equivalent definition.

Next, we define the SSS-security of PO. Consider game SSS of Fig. $\mathbb{\nabla}$ associated to a pointfunction obfuscator Obf, a target generator $\mathrm{X}$, an adversary $\mathcal{A}$, a simulator $\mathcal{S}$ and a predicate 


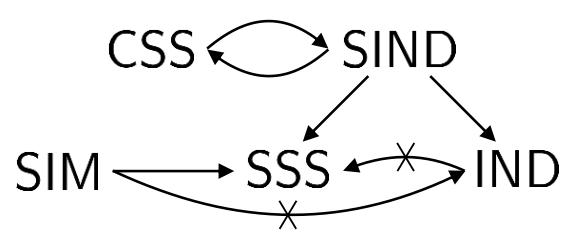

Figure 9: Relations between security notions for point-function obfuscation.

algorithm $\mathcal{P}$, such that Obf.tI $=\mathrm{X} . \mathrm{tl}$ and algorithms $\mathcal{A}, \mathcal{S}, \mathcal{P}$ are required to return outputs in $\{0,1\}$. For $\lambda \in \mathbb{N}$ let $\operatorname{Adv}_{\mathrm{Obf}, \mathbf{X}, \mathcal{A}, \mathcal{S}, \mathcal{P}}^{\mathrm{sss}}(\lambda)=2 \operatorname{Pr}\left[\operatorname{SSS}_{\mathrm{Obf}, \mathbf{X}}^{\mathcal{A}, \mathcal{S}, \mathcal{P}}(\lambda)\right]-1$. Let $\mathbf{X}$ be a class of target generators. We say that Obf is $\operatorname{SSS}[\mathbf{X}]$-secure if for every target generator $\mathrm{X} \in \mathbf{X}$ and every PT $\mathcal{A}$ there exists PT $\mathcal{S}$ such that $\operatorname{Adv}_{\mathrm{Obf}, X, \mathcal{A}, \mathcal{S}, \mathcal{P}}^{\text {sss }}(\cdot)$ is negligible for every $\mathrm{PT} \mathcal{P}$. The notion of SSS-security is weaker than that of SIM-security because in the former $X$ is used to randomly sample target points, whereas the latter quantifies over every $(\mathbf{k}, a) \in\left[\mathrm{X} \cdot \operatorname{Ev}\left(1^{\lambda}\right)\right]$. In contrast to SIM-security, predicate $\mathcal{P}$ in the definition of SSS-security can not be substituted with a constant function to achieve an equivalent definition. Namely, replacing $\mathcal{P}$ with a constant function would allow $\mathcal{S}$ to run $\mathrm{X}$ in order to generate fresh $(\mathbf{k}, a)$, obfuscate $\mathbf{I}_{\mathbf{k}}$ to get $\overline{\mathbf{P}}$, and simulate $\mathcal{A}$ on $\overline{\mathbf{P}}, a$. As a result, every obfuscator would be vaciously SSS-secure for any class of target generators $\mathbf{X}$.

There is a number of further definitional choices for simulation-based security notions. We briefly mention the three most common ones. First, the order of quantification that we use can be changed to require weaker security. Informally, our current definitions require that for every adversary $\mathcal{A}$ there exists a simulator $\mathcal{S}$ such that the advantage of the corresponding game is negligible. Definitions using this quantification are sometimes called strong and are used in [41, 51, 35, [2.9, 26, [14, 6]. In contrast, a weak definition would require that for every adversary $\mathcal{A}$ and every polynomial $p$ there exists a simulator $\mathcal{S}$ such that the advantage is bounded by $1 / p$. Such definitions are used in [23, 25, 51, 35, 42, 26]. Second, Goldwasser and Kalai [35] formalized two variants of auxiliary information that is given to the adversary - dependent and independent - meaning that either the auxiliary information is allowed to depend on the circuit being obfuscated, or it is not allowed to. Some of the prior work [35, 42] considers both dependent and independent auxiliary information, whereas [2:3, 2.9] define point-function obfuscation only for dependent auxiliary information. Third, Bitansky and Canetti [II] introduce the notion of VGBO (Virtual Grey Box Obfuscation), which relaxes the simulation-based security by considing unbounded adversaries (that are still allowed to make only a polynomial number of oracle queries).

RELATIONS BETWEEN SECURITY NOTIONS. Fig. 1 shows relations between the security notions for point-function obfuscation discussed in this paper. Consider any two security notions A and B. An arrow from $\mathrm{A}$ to $\mathrm{B}$ means that any $\mathrm{A}[\mathbf{X}]$-secure point-function obfuscator is also $\mathrm{B}[\mathbf{X}]$-secure, for every class of target generators $\mathbf{X}$. A crossed arrow going from A to B means that there exists an obfuscator Obf and a class of target generators $\mathbf{X}$ such that Obf is $\mathrm{A}[\mathbf{X}]$-secure but not $\mathrm{B}[\mathbf{X}]$-secure.

Implications SIM $\rightarrow$ SSS and SIND $\rightarrow$ IND trivially follow from our definitions of the corresponding security notions. The proofs for all other implications and separations that are shown in Fig. 9 are provided below. Our relations do not answer whether any of SSS and CSS imply SIM; we leave these as open problems. All other implications and separations between any two security notions can be deduced using transitivity of the relations in Fig. Q 0 .

Theorem 6.1 There exists a point-function obfuscator Obf and a class of target generators $\mathbf{X}$ such that (a) Obf is $\operatorname{IND}[\mathbf{X}]$-secure, and (b) Obf is not $\mathrm{SSS}[\mathbf{X}]$-secure. 
Proof of Theorem [6.7: We build an obfuscator Obf and a target generator $\mathbf{X}$ as follows. Let $\mathrm{X} . v \mathrm{l}, \mathrm{X} . \mathrm{tl}: \mathbb{N} \rightarrow \mathbb{N}$ and Obf.tl $=\mathrm{X} . \mathrm{tl}$, such that $2^{-\mathrm{X} . \mathrm{tl}(\cdot)}$ is negligible. For any $\lambda \in \mathbb{N}$ let

$$
\begin{array}{l|l}
\text { Obfuscator } \operatorname{Obf}\left(1^{\lambda}, \mathbf{I}_{\mathbf{k}}\right) & \begin{array}{l}
\text { Algorithm } \mathbf{X} \cdot \operatorname{Ev}\left(1^{\lambda}\right) \\
\text { Return } \mathbf{I}_{\mathbf{k}}
\end{array} \\
\begin{array}{l}
\text { For } i=1, \ldots, \mathrm{X} \cdot \mathrm{vl}(\lambda) \\
\text { Return }(\mathbf{k}, \varepsilon)
\end{array}
\end{array}
$$

Let $\mathbf{X}=\{\mathbf{X}\}$. Target generator $\mathbf{X}$ samples targets from a uniformly random distribution and returns an empty string as auxiliary information, so $\mathbf{X}$ is trivial and $\mathbf{X} \subseteq \mathbf{X}^{\text {triv }}$. It follows from Theorem $[$.$] that Obf is \operatorname{IND}[\mathbf{X}]$-secure.

We now argue that Obf is not $\operatorname{SSS}[\mathbf{X}]$-secure. Consider a $\mathrm{PT}$ adversary $\mathcal{A}$ and a $\mathrm{PT}$ predicate algorithm $\mathcal{P}$ defined as follows. For any $\lambda \in \mathbb{N}$ let

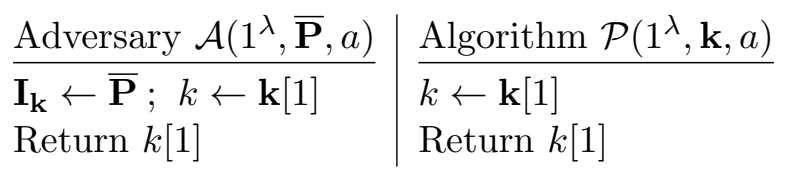

Both $\mathcal{A}$ and $\mathcal{P}$ always return the first bit of target point $\mathbf{k}[1]$. Then for every PT simulator $\mathcal{S}$, algorithms $\mathcal{A}$ and $\mathcal{P}$ in game $\operatorname{SSS}_{\mathrm{Obf}, \mathrm{X}}^{\mathcal{A}, \mathcal{S}}(\lambda)$ produce the same output with probability 1 . But there does not exist a simulator $\mathcal{S}$ that returns the first bit of $\mathbf{k}[1]$ with the same probability, assuming that $\mathrm{X}$ is unpredictable, which follows from the assumption that $2^{-\mathrm{X} . \mathrm{t}(\cdot)}$ is negligible. Therefore, we can conclude that Obf is not $\mathrm{SSS}[\mathbf{X}]$-secure.

Theorem 6.2 There exists a point-function obfuscator Obf and a class of target generators $\mathbf{X}$ such that (a) Obf is $\mathrm{SIM}[\mathbf{X}]$-secure, and (b) Obf is not $\operatorname{IND}[\mathbf{X}]$-secure.

Proof of Theorem 6.2: We build an obfuscator Obf and a target generator $X$ as follows. Let X.vl,X.tl: $\mathbb{N} \rightarrow \mathbb{N}$ and Obf.tl $=$ X.tl. For any $\lambda \in \mathbb{N}$ let

$$
\begin{array}{l|l}
\text { Obfuscator } \operatorname{Obf}\left(1^{\lambda}, \mathbf{I}_{\mathbf{k}}\right) & \begin{array}{l}
\text { Algorithm X.Ev }\left(1^{\lambda}\right) \\
\text { Return } \mathbf{I}_{\mathbf{k}}
\end{array} \\
\begin{array}{l}
\text { For } i=1, \ldots, \mathrm{X} \cdot \mathrm{vl}(\lambda) \\
\text { Return }(\mathbf{k}, \mathbf{k})
\end{array}
\end{array} \text { do } \mathbf{k}[i] \leftarrow \$\{0,1\}^{\mathrm{X} \cdot \mathrm{tl}(\lambda)}
$$

Let $\mathbf{X}=\{\mathbf{X}\}$.

First, we show that Obf is $\operatorname{SIM}[\mathbf{X}]$-secure. Let $\mathcal{A}$ be a PT adversary attacking the $\operatorname{SIM}[\mathbf{X}]$-security of Obf. We build a PT simulator $\mathcal{S}$ as follows:

$$
\begin{aligned}
& \frac{\text { Simulator } \mathcal{S}^{\mathbf{I}_{\mathbf{k}}}\left(1^{\lambda}, a\right)}{\mathbf{k} \leftarrow a ; \overline{\mathbf{P}} \leftarrow \$ \operatorname{Obf}\left(1^{\lambda}, \mathbf{I}_{\mathbf{k}}\right)} \\
& p \leftarrow \$ \mathcal{A}\left(1^{\lambda}, \overline{\mathbf{P}}, a\right) ; \text { Return } p
\end{aligned}
$$

The simulator $\mathcal{S}$ produces the same output distribution as $\mathcal{A}$, so Obf is $\operatorname{SIM}[\mathbf{X}]$-secure.

Next, we show that Obf is not $\operatorname{IND}[\mathbf{X}]$-secure. We build a PT adversary $\mathcal{B}$ attacking the $\operatorname{IND}[\mathbf{X}]$ security of Obf as follows: 


\begin{tabular}{|c|}
\hline Games $\mathrm{G}_{0}, \mathrm{G}_{1}$ \\
\hline$b \leftarrow s\{0,1\} ; \quad\left(\mathbf{k}_{1}, a_{1}\right) \leftarrow s \mathrm{X} \cdot \operatorname{Ev}\left(1^{\lambda}\right)$ \\
\hline For $i=1, \ldots, \mathrm{X} \cdot \mathrm{vl}(\lambda)$ do $\mathbf{k}_{0}[i] \leftarrow \$\{0,1\}^{\mathrm{X} . \mathrm{tl}(\lambda)}$ \\
\hline$\overline{\mathbf{P}} \leftarrow_{\S} \operatorname{Obf}\left(1^{\lambda}, \mathbf{I}_{\mathbf{k}_{b}}\right) ; d \leftarrow \AA \mathcal{A}\left(1^{\lambda}, \overline{\mathbf{P}}, a_{1}\right)$ \\
\hline$b_{0} \leftarrow \$ \mathcal{D}\left(1^{\lambda}, \mathbf{k}_{1}, a_{1}, 0\right) ; b_{1} \leftarrow \$ \mathcal{D}\left(1^{\lambda}, \mathbf{k}_{1}, a_{1}, 1\right)$ \\
\hline If $\left(b_{0}=b_{1}\right)$ then $b^{\prime} \leftarrow b_{0}$ else $b^{\prime} \leftarrow b_{d} \quad / / \mathrm{G}_{0}$ \\
\hline If $\left(b_{0}=b_{1}\right)$ then $b^{\prime} \leftarrow \$\{0,1\}$ else $b^{\prime} \leftarrow b_{d} \quad / / \mathrm{G}_{1}$ \\
\hline Return $\left(b=b^{\prime}\right)$ \\
\hline
\end{tabular}

Figure 10: Games for proof of Theorem [6.3.

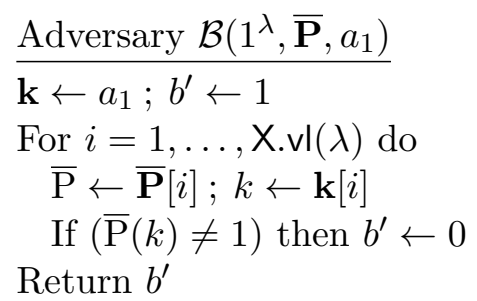

Adversary $\mathcal{B}$ uses target vector $\mathbf{k}$ that is leaked through the auxiliary information in order to check whether $\overline{\mathbf{P}}$ is an obfuscation of $\mathbf{I}_{\mathbf{k}}$. For any $\lambda \in \mathbb{N}$ we have $\operatorname{Adv}_{\mathrm{Obf}, \mathrm{X}, \mathcal{B}}^{\text {ind }}(\lambda)=1-2^{-\mathrm{X} \cdot \mathrm{vl}(\lambda) \cdot \mathrm{X} \cdot \mathrm{tl}(\lambda)}$, where $2^{-\mathrm{X} . \mathrm{vl}(\lambda) \cdot \mathrm{X} \cdot \mathrm{tl}(\lambda)}$ is the probability that $\mathbf{k}_{0}[i]=\mathbf{k}_{1}[i]$ for every $i \in\{1, \ldots, \mathrm{X} \cdot \mathrm{vl}(\lambda)\}$ in game $\operatorname{IND}_{\mathrm{Obf}, \mathrm{X}}^{\mathcal{B}}(\lambda)$.

Theorem 6.3 Let Obf be a point-function obfuscator. Let $\mathbf{X}$ be a class of target generators. Assume that $\mathrm{Obf}$ is $\mathrm{CSS}[\mathbf{X}]$-secure. Then $\mathrm{Obf}$ is $\operatorname{SIND}[\mathbf{X}]$-secure.

Proof of Theorem 6.3: $\quad$ Let $\mathbf{X} \in \mathbf{X}$ be a target generator. Let $\mathcal{A}$ be a PT adversary and let $\mathcal{D}$ be a PT distinguisher, both attacking the SIND-security of Obf with respect to $\mathrm{X}$. Consider the games of Fig. W. Lines not annotated with comments are common to both games. Game $\mathrm{G}_{0}$ is equivalent to game $\operatorname{SIND}_{\mathrm{Obf}, \mathrm{X}}^{\mathcal{A}, \mathcal{D}}(\lambda)$, so we have $\operatorname{Adv}_{\mathrm{Obf}, \mathrm{X}, \mathcal{A}, \mathcal{D}}^{\text {sind }}(\lambda)=2 \operatorname{Pr}\left[\mathrm{G}_{0}\right]-1$.

We claim that $\operatorname{Pr}\left[\mathrm{G}_{1}\right]=\operatorname{Pr}\left[\mathrm{G}_{0}\right]$. This is true because $\mathrm{G}_{0}$ and $\mathrm{G}_{1}$ differ only in the choice of $b^{\prime}$ for the case when $b_{0}=b_{1}$, meaning that both games produce $b^{\prime}$ independently of the challenge bit $b$.

Next, we construct a PT adversary $\mathcal{B}=\left(\mathcal{B}_{1}, \mathcal{B}_{2}\right)$ attacking the CSS-security of Obf with respect to $\mathrm{X}$, as follows:

\begin{tabular}{l|l} 
Adversary $\mathcal{B}_{1}\left(1^{\lambda}, \mathbf{k}_{1}, a_{1}\right)$ & Adversary $\mathcal{B}_{2}\left(1^{\lambda}, \overline{\mathbf{P}}, a_{1}\right)$ \\
\hline$b_{0} \leftarrow \$ \mathcal{D}\left(1^{\lambda}, \mathbf{k}_{1}, a_{1}, 0\right) ; b_{1} \leftarrow \$ \mathcal{D}\left(1^{\lambda}, \mathbf{k}_{1}, a_{1}, 1\right)$ & $t^{\prime} \leftarrow \mathcal{A}\left(1^{\lambda}, \overline{\mathbf{P}}, a_{1}\right)$ \\
If $\left(b_{0}=b_{1}\right)$ then $t \leftarrow \$\{0,1\}$ & Return $t^{\prime}$ \\
Else if $\left(b_{1}=1\right)$ then $t \leftarrow 1$ & \\
Else if $\left(b_{0}=1\right)$ then $t \leftarrow 0$ & \\
Return $t$ &
\end{tabular}

We claim that $\operatorname{Pr}\left[\mathrm{G}_{1}\right]=\operatorname{Pr}\left[\operatorname{CSS}_{\text {Obf }, \mathrm{X}}^{\mathcal{B}}(\lambda)\right]$. Specifically, we show that adversary $\mathcal{B}_{1}$ returns $t \in\{0,1\}$ such that evaluating

$$
\text { If }\left(b_{0}=b_{1}\right) \text { then } b^{\prime} \leftarrow \$\{0,1\} \text { else } b^{\prime} \leftarrow b_{d}
$$


in game $\mathrm{G}_{1}$ produces the same distribution over $b^{\prime} \in\{0,1\}$ as evaluating

$$
\text { If }\left(t=t^{\prime}\right) \text { then } b^{\prime} \leftarrow 1 \text { else } b^{\prime} \leftarrow 0
$$

in game $\operatorname{CSS}_{\mathrm{Obf}, \mathrm{X}}^{\mathcal{B}}(\lambda)$. For this purpose, consider game $\mathrm{G}_{1}$. If $b_{0}=b_{1}$ then a random bit is assigned to $b^{\prime}$ in $\mathrm{G}_{1}$; adversary $\mathcal{B}_{1}$ achieves the same in $\operatorname{CSS}_{\text {Obf,X }}^{\mathcal{B}}(\lambda)$ by precomputing the values of $b_{0}$ and $b_{1}$ and assigning a random bit to $t$ if $b_{0}=b_{1}$. Next, if $b_{0} \neq b_{1}$ in $\mathrm{G}_{1}$ then $b_{d}$ is assigned to $b^{\prime}$; adversary $\mathcal{B}_{1}$ achieves the same in $\operatorname{CSS}_{\text {Obf }, \mathrm{X}}^{\mathcal{B}}(\lambda)$ by returning the unique value $t \in\{0,1\}$ such that $b_{t}=1$.

Combining the above equations, we get

$$
\operatorname{Adv}_{\mathrm{Obf}, \mathrm{X}, \mathcal{A}, \mathcal{D}}^{\text {sind }}(\lambda)=2 \operatorname{Pr}\left[\mathrm{G}_{0}\right]-1=2 \operatorname{Pr}\left[\mathrm{G}_{1}\right]-1=2 \operatorname{Pr}\left[\operatorname{CSS}_{\mathrm{Obf}, \mathrm{X}}^{\mathcal{B}}(\lambda)\right]-1=\operatorname{Adv}_{\mathrm{Obf}, \mathrm{X}, \mathcal{B}}^{\mathrm{css}}(\lambda) .
$$

for all $\lambda \in \mathbb{N}$.

Theorem 6.4 Let Obf be a point-function obfuscator. Let $\mathbf{X}$ be a class of target generators. Assume that $\mathrm{Obf}$ is $\mathrm{SIND}[\mathbf{X}]$-secure. Then $\mathrm{Obf}$ is $\mathrm{CSS}[\mathbf{X}]$-secure.

Proof of Theorem 6.4: Let $\mathrm{X} \in \mathbf{X}$ be a target generator. Let $\mathcal{A}=\left(\mathcal{A}_{1}, \mathcal{A}_{2}\right)$ be a PT adversary attacking the CSS-security of Obf with respect to $\mathrm{X}$. We construct a PT adversary $\mathcal{B}$ and a PT distinguisher $\mathcal{D}$ attacking the SIND-security of Obf with respect to $\mathrm{X}$, as follows:

$$
\begin{array}{l|l}
\frac{\text { Adversary } \mathcal{B}\left(1^{\lambda}, \overline{\mathbf{P}}, a_{1}\right)}{t^{\prime} \leftarrow \$ \mathcal{A}_{2}\left(1^{\lambda}, \overline{\mathbf{P}}, a_{1}\right)} & \frac{\text { Distinguisher } \mathcal{D}\left(1^{\lambda}, \mathbf{k}_{1}, a_{1}, t^{\prime}\right)}{t \leftarrow \$ \mathcal{A}_{1}\left(1^{\lambda}, \mathbf{k}_{1}, a_{1}\right)} \\
\text { return } t^{\prime} & \begin{array}{l}
\text { If }\left(t=t^{\prime}\right) \text { then } b^{\prime} \leftarrow 1 \text { else } b^{\prime} \leftarrow 0 \\
\text { Return } b^{\prime}
\end{array}
\end{array}
$$

We have $\operatorname{Pr}\left[\operatorname{SiND}_{\mathrm{Obf}, \mathbf{X}}^{\mathcal{B}, \mathcal{D}}(\lambda)\right]=\operatorname{Pr}\left[\operatorname{CSS}_{\mathrm{Obf}, \mathrm{X}}^{\mathcal{A}}(\lambda)\right]$ for all $\lambda \in \mathbb{N}$ According to the assumed $\operatorname{SIND}[\mathbf{X}]$ security of Obf, it follows that $\operatorname{Adv}_{\mathrm{Obf}, \mathrm{X}, \mathcal{A}}^{\mathrm{css}}(\cdot)$ is negligible.

Theorem 6.5 Let Obf be a point-function obfuscator. Let $\mathbf{X}$ be a class of target generators. Assume that $\mathrm{Obf}$ is $\mathrm{SIND}[\mathbf{X}]$-secure. Then $\mathrm{Obf}$ is $\mathrm{SSS}[\mathbf{X}]$-secure.

Proof of Theorem 6.5: Let $\mathrm{X} \in \mathbf{X}$ be a target generator. Let $\mathcal{A}$ be a PT adversary attacking the SSS-security of Obf with respect to $\mathrm{X}$. We construct the corresponding PT simulator $\mathcal{S}$ as follows:

$$
\begin{aligned}
& \underline{\text { Simulator } \mathcal{S}^{\mathbf{I}_{\mathbf{k}}}\left(1^{\lambda}, a_{1}\right)} \\
& \text { For } i=1, \ldots, \mathrm{X} . v \mathrm{vl}(\lambda) \text { do } \mathbf{k}_{0}[i] \leftarrow \$\{0,1\}^{\mathrm{X} . \mathrm{tl}(\lambda)} \\
& \overline{\mathbf{P}} \leftarrow{ }_{\$} \operatorname{Obf}\left(1^{\lambda}, \mathbf{I}_{\mathbf{k}_{0}}\right) ; p^{\prime} \leftarrow \$ \mathcal{A}\left(1^{\lambda}, \overline{\mathbf{P}}, a_{1}\right) \\
& \text { Return } p^{\prime}
\end{aligned}
$$

Let $\mathcal{P}$ be a PT predicate algorithm. We construct a PT adversary $\mathcal{B}$ and a PT distinguisher $\mathcal{D}$ attacking the SIND-security of Obf with respect to $\mathrm{X}$, as follows:

$$
\begin{array}{l|l}
\frac{\text { Adversary } \mathcal{B}\left(1^{\lambda}, \overline{\mathbf{P}}, a_{1}\right)}{p^{\prime} \leftarrow \$ \mathcal{A}\left(1^{\lambda}, \overline{\mathbf{P}}, a_{1}\right)} & \frac{\text { Distinguisher } \mathcal{D}\left(1^{\lambda}, \mathbf{k}_{1}, a_{1}, p^{\prime}\right)}{p \leftarrow \$ \mathcal{P}\left(1^{\lambda}, \mathbf{k}_{1}, a_{1}\right)} \\
\text { Return } p^{\prime} & \begin{array}{l}
\text { If }\left(p=p^{\prime}\right) \text { then } b^{\prime} \leftarrow 1 \text { else } b^{\prime} \leftarrow 0 \\
\text { Return } b^{\prime}
\end{array}
\end{array}
$$


We have $\operatorname{Pr}\left[\operatorname{SIND}_{\mathrm{Obf}, \mathbf{X}}^{\mathcal{B}, \mathcal{D}}(\lambda)\right]=\operatorname{Pr}\left[\operatorname{SSS}_{\text {Obf,X }}^{\mathcal{A}, \mathcal{S}, \mathcal{P}}(\lambda)\right]$ for all $\lambda \in \mathbb{N}$. According to the assumed $\operatorname{SIND}[\mathbf{X}]$ security of Obf, it follows that $\operatorname{Adv}_{\mathrm{Obf}, \mathrm{X}, \mathcal{A}, \mathcal{S}, \mathcal{P}}(\cdot)$ is negligible.

Security of our PO Constructions. Let $\mathbf{X}$ be a class of target generators. In Section $\mathbf{5}$ we showed how to build a point-function obfuscator that is $\operatorname{IND}[\mathbf{X}]$-secure, based on any of the following: an injective OWF $[\mathbf{X}]$-secure function family and an $\mathrm{iO}$, or a PRIV1 $[\mathbf{X}]$-secure DPKE, or an $\operatorname{mUCE}\left[\mathbf{S}^{\mathbf{X}}\right]$-secure function family, for $\mathbf{S}^{\mathbf{X}}$ as defined in Section 5.3 . We do not know how to adapt our constructions to achieve SIM $[\mathbf{X}]$-security. But each of our construction achieves CSS[X]security, requiring only minimal changes in the used assumptions.

We now provide some intuition about our claim. Recall that game CSS computes $t \in\{0,1\}$ by running $\mathcal{A}_{1}\left(1^{\lambda}, \mathbf{k}_{1}, a_{1}\right)$, and subsequently compares it to the output of $\mathcal{A}_{2}\left(1^{\lambda}, \overline{\mathbf{P}}, a_{1}\right)$. This is different from game IND where the adversary consists only of an algorithm $\mathcal{A}\left(1^{\lambda}, \overline{\mathbf{P}}, a_{1}\right)$. The difficulty of adapting proofs of $\operatorname{IND}[\mathbf{X}]$-security to achieve $\operatorname{CSS}[\mathbf{X}]$-security is that in the latter $\mathbf{k}_{1}$ (required to run $\mathcal{A}_{1}$ ) and $\overline{\mathbf{P}}$ (required to run $\mathcal{A}_{2}$ ) are usually available in different stages of the security proof, meaning that one has to find a way to pass around the value of $t$ (which depends on $\mathbf{k}_{1}$ ) across the stages. We resolve this by pushing $t$ into the auxiliary information of target generators that parametrize our security notions.

Let $\mathbf{X}$ be a class of target generators. Let $\mathbf{P}$ be the set of all PT predicate algorithms $\mathcal{P}$ such that $\mathcal{P}\left(1^{\lambda}, \cdot, \cdot\right):\{0,1\}^{*} \times\{0,1\}^{*} \rightarrow\{0,1\}$ for all $\lambda \in \mathbb{N}$. For any $\lambda \in \mathbb{N}, \mathbf{X} \in \mathbf{X}$ and $\mathcal{P} \in \mathbf{P}$ let $\mathbf{X}^{\mathcal{P}}$ be defined as follows:

$$
\begin{aligned}
& \frac{\text { Source } \mathbf{X}^{\mathcal{P}}\left(1^{\lambda}\right)}{(\mathbf{k}, a) \leftarrow \$ \mathrm{X}\left(1^{\lambda}\right)} \\
& \beta \leftarrow \$ \mathcal{P}\left(1^{\lambda}, \mathbf{k}, a\right) \\
& \text { Return }(\mathbf{k},(a, \beta))
\end{aligned}
$$

where $X^{\mathcal{P}} . v l=X . v l$ and $X^{\mathcal{P}} . t l=X . t l$. We define a new class of target generators $\mathbf{X}^{\prime}=\left\{X^{\mathcal{P}}: \mathbf{X} \in\right.$ $\mathbf{X}, \mathcal{P} \in \mathbf{P}\}$. Then each of our constructions from Section $\mathbf{5}$ achieves CSS $[\mathbf{X}]$-security, based on either of the following: an injective $\mathrm{OWF}\left[\mathbf{X}^{\prime}\right]$-secure function family and an $\mathrm{iO}$, or a PRIV1 $\left[\mathbf{X}^{\prime}\right]$-secure DPKE, or an mUCE $\left[\mathbf{S}^{\mathbf{X}^{\prime}}\right]$-secure function family.

Note that for any $\mathbf{X} \in \mathbf{X}$ and $\mathcal{P} \in \mathbf{P}$, the construction of $\mathbf{X}^{\mathcal{P}}$ expands the auxiliary information of $\mathrm{X}$ only by a single bit. This means that $\mathrm{X}^{\mathcal{P}}$ inherits the unpredictability properties of $\mathrm{X}$. Namely, for any $\lambda \in \mathbb{N}, \mathbf{X} \in \mathbf{X}, \mathcal{P} \in \mathbf{P}$ and any PT adversary $\mathcal{R}$ we can construct a PT adversary $\mathcal{Q}$ such that $\operatorname{Pr}\left[\operatorname{PRED}_{\mathbf{X}^{\mathcal{Q}}}^{\mathcal{Q}}(\lambda)\right] \geq \frac{1}{2} \operatorname{Pr}\left[\operatorname{PRED}_{\mathrm{X}^{\mathcal{P}}}^{\mathcal{R}}(\lambda)\right]$ for all $\lambda \in \mathbb{N}$. Adversary $\mathcal{Q}$ would attempt to guess the extra bit of information and then simulate $\mathcal{R}$. The same approach can be used to show that any $\mathrm{OWF}[\mathbf{X}]$-secure function family is also $\mathrm{OWF}\left[\mathbf{X}^{\prime}\right]$-secure, recoving the construction of $\mathrm{CSS}[\mathbf{X}]$-secure $\mathrm{PO}$ directly from a $\mathrm{OWF}[\mathbf{X}]$-secure function family and an $\mathrm{iO}$.

MULTI-BIT OUTPUTS IN SECURITY GAMES. Informally, simulation-based security definitions require that for any predicate $\mathcal{P}$ : if $\mathcal{P}(\mathbf{k})$ can be computed from an obfusaction of $\mathbf{I}_{\mathbf{k}}$, then it can also be computed from just an input-output access to $\mathbf{I}_{\mathbf{k}}$. These definitions can be extended to also consider multi-bit properties of $\mathbf{k}$ that can be computed from $\mathbf{I}_{\mathbf{k}}$, meaning that the above would be required to hold for any function $\mathcal{F}$, and not just for any predicate $\mathcal{P}$. Our definition of CSS security can be extended in the same way. Doing this would likely change some of our implications from Fig. Q1. In particular, note that our definitions of CSS and SSS are similar to those that were used for DPKE schemes in BFOR [G], who showed them to be equivalent for multiple-bit outputs in their setting. It is an open problem to study the security notions of obfuscation that allow multi-bit outputs.

Barak et al. [2] considered multiple ways to define the security of VBB obfuscation, and chose the weakest, predicate-based definition in order to achieve a stronger impossibility result. Wee [5T] 
showed that the strongest definitional variant of [2], requiring that the outputs of adverasary and simulator are computationally indistinguishable, is only achievable for classes of functions that are efficiently and exactly learnable using membership queries. (Wee's analysis was for the case when no auxiliary information is available.) However, function-based and relation-based definitions of [2] are not well-studied. The definitions of average-case simulation security for obfuscation of general functions [3., 38] do consider adversaries and simulators that are allowed to return multibit outputs, but most of the work on point-function obfuscation continues to use predicate-based definitions.

\section{Acknowledgments}

We thank Ran Canetti, Arno Mittelbach and Stefano Tessaro for discussions and insights. Extensive and insightful comments by the TCC 2016-A reviewers lead to considerable changes and additions to the paper including Theorem B.], Corollary $\mathbf{5 . 4}$ and Section 6 .

\section{References}

[1] P. Ananth, D. Boneh, S. Garg, A. Sahai, and M. Zhandry. Differing-inputs obfuscation and applications. Cryptology ePrint Archive, Report 2013/689, 2013. http://eprint.iacr.org/2013/689. 田, [, \&

[2] B. Barak, O. Goldreich, R. Impagliazzo, S. Rudich, A. Sahai, S. P. Vadhan, and K. Yang. On the (im)possibility of obfuscating programs. In J. Kilian, editor, CRYPTO 2001, volume 2139 of LNCS,

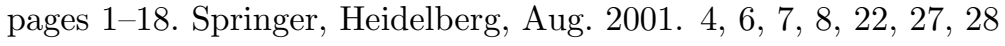

[3] M. Bellare, A. Boldyreva, and A. O'Neill. Deterministic and efficiently searchable encryption. In A. Menezes, editor, CRYPTO 200\%, volume 4622 of LNCS, pages 535-552. Springer, Heidelberg, Aug. 2007. [1, 回, [7, 미, 미, [2]

[4] M. Bellare, M. Fischlin, A. O'Neill, and T. Ristenpart. Deterministic encryption: Definitional equivalences and constructions without random oracles. In D. Wagner, editor, CRYPTO 2008, volume 5157

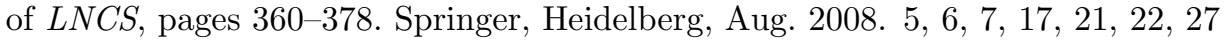

[5] M. Bellare and V. T. Hoang. Resisting randomness subversion: Fast deterministic and hedged publickey encryption in the standard model. In E. Oswald and M. Fischlin, editors, EUROCRYPT 2015, Part II, volume 9057 of LNCS, pages 627-656. Springer, Heidelberg, Apr. 2015. 回, 미, 피

[6] M. Bellare, V. T. Hoang, and S. Keelveedhi. Instantiating random oracles via UCEs. Cryptology ePrint

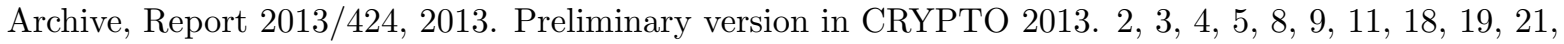
[2.2, 20.3

[7] M. Bellare, V. T. Hoang, and S. Keelveedhi. Instantiating random oracles via UCEs. In R. Canetti and J. A. Garay, editors, CRYPTO 2013, Part II, volume 8043 of LNCS, pages 398-415. Springer, Heidelberg, Aug. 2013. \$

[8] M. Bellare and P. Rogaway. The security of triple encryption and a framework for code-based gameplaying proofs. In S. Vaudenay, editor, EUROCRYPT 2006, volume 4004 of LNCS, pages 409-426. Springer, Heidelberg, May / June 2006. [

[9] M. Bellare, I. Stepanovs, and S. Tessaro. Poly-many hardcore bits for any one-way function and a framework for differing-inputs obfuscation. In P. Sarkar and T. Iwata, editors, ASIACRYPT 2014, Part II, volume 8874 of LNCS, pages 102-121. Springer, Heidelberg, Dec. 2014. 田, $\mathbf{0}$

[10] M. Bellare, I. Stepanovs, and S. Tessaro. Contention in cryptoland: Obfuscation, leakage and uce. In Theory of Cryptography, TCC 2016-A. Springer, 2016. [2, 20] 
[11] N. Bitansky and R. Canetti. On strong simulation and composable point obfuscation. In T. Rabin, editor, CRYPTO 2010, volume 6223 of LNCS, pages 520-537. Springer, Heidelberg, Aug. 2010. ㅁ, [6, [2], [2], [2.]

[12] N. Bitansky, R. Canetti, Y. T. Kalai, and O. Paneth. On virtual grey box obfuscation for general circuits. In J. A. Garay and R. Gennaro, editors, CRYPTO 2014, Part II, volume 8617 of LNCS, pages 108-125. Springer, Heidelberg, Aug. 2014. [2, 田

[13] N. Bitansky, R. Canetti, O. Paneth, and A. Rosen. On the existence of extractable one-way functions. In D. B. Shmoys, editor, 46th ACM STOC, pages 505-514. ACM Press, May / June 2014. [1, ㅁ.

[14] N. Bitansky and O. Paneth. Point obfuscation and 3-round zero-knowledge. In R. Cramer, editor,

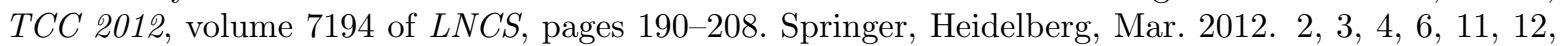
[2], [22, [2.]

[15] A. Boldyreva, S. Fehr, and A. O'Neill. On notions of security for deterministic encryption, and efficient constructions without random oracles. In D. Wagner, editor, CRYPTO 2008, volume 5157 of LNCS, pages 335-359. Springer, Heidelberg, Aug. 2008. 回, 띠, ㄸ

[16] E. Boyle, K.-M. Chung, and R. Pass. On extractability obfuscation. In Y. Lindell, editor, TCC 2014,

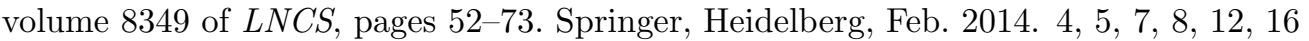

[17] Z. Brakerski and G. Segev. Better security for deterministic public-key encryption: The auxiliaryinput setting. In P. Rogaway, editor, CRYPTO 2011, volume 6841 of LNCS, pages 543-560. Springer,

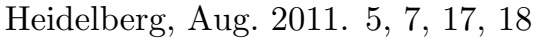

[18] C. Brzuska, P. Farshim, and A. Mittelbach. Indistinguishability obfuscation and UCEs: The case of computationally unpredictable sources. In J. A. Garay and R. Gennaro, editors, CRYPTO 2014, Part

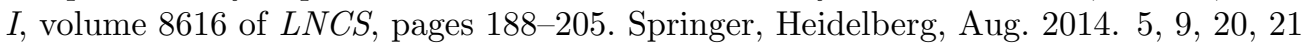

[19] C. Brzuska, P. Farshim, and A. Mittelbach. Random-oracle uninstantiability from indistinguishability obfuscation. In Y. Dodis and J. B. Nielsen, editors, TCC 2015, Part II, volume 9015 of LNCS, pages 428-455. Springer, Heidelberg, Mar. 2015. 22]

[20] C. Brzuska and A. Mittelbach. Indistinguishability obfuscation versus multi-bit point obfuscation with auxiliary input. In P. Sarkar and T. Iwata, editors, ASIACRYPT 2014, Part II, volume 8874 of LNCS,

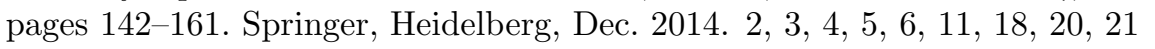

[21] C. Brzuska and A. Mittelbach. Using indistinguishability obfuscation via UCEs. In P. Sarkar and T. Iwata, editors, ASIACRYPT 2014, Part II, volume 8874 of LNCS, pages 122-141. Springer, Heidel-

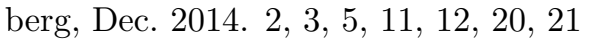

[22] C. Brzuska and A. Mittelbach. Universal computational extractors and the superfluous padding assumption for indistinguishability obfuscation. Cryptology ePrint Archive, Report 2015/581, 2015.

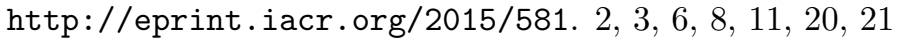

[23] R. Canetti. Towards realizing random oracles: Hash functions that hide all partial information. In B. S. Kaliski Jr., editor, CRYPTO'97, volume 1294 of LNCS, pages 455-469. Springer, Heidelberg, Aug. 1997.

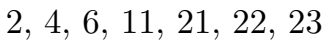

[24] R. Canetti and R. R. Dakdouk. Extractable perfectly one-way functions. In L. Aceto, I. Damgård, L. A. Goldberg, M. M. Halldórsson, A. Ingólfsdóttir, and I. Walukiewicz, editors, ICALP 2008, Part

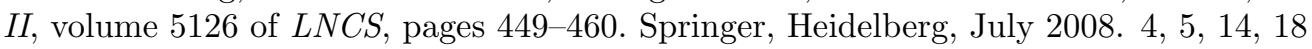

[25] R. Canetti and R. R. Dakdouk. Obfuscating point functions with multibit output. In N. P. Smart, editor, EUROCRYPT 2008, volume 4965 of LNCS, pages 489-508. Springer, Heidelberg, Apr. 2008. ㄴ,

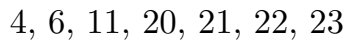

[26] R. Canetti, Y. T. Kalai, M. Varia, and D. Wichs. On symmetric encryption and point obfuscation. In D. Micciancio, editor, TCC 2010, volume 5978 of LNCS, pages 52-71. Springer, Heidelberg, Feb. 2010. [2, [2], [2], [2:3] 
[27] R. Canetti, D. Micciancio, and O. Reingold. Perfectly one-way probabilistic hash functions (preliminary

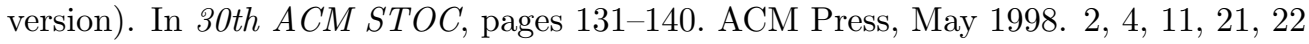

[28] Y. Dodis, C. Ganesh, A. Golovnev, A. Juels, and T. Ristenpart. A formal treatment of backdoored pseudorandom generators. In E. Oswald and M. Fischlin, editors, EUROCRYPT 2015, Part I, volume 9056 of LNCS, pages 101-126. Springer, Heidelberg, Apr. 2015.

[29] Y. Dodis, Y. T. Kalai, and S. Lovett. On cryptography with auxiliary input. In M. Mitzenmacher,

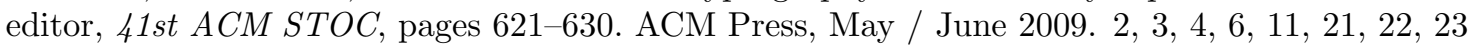

[30] Y. Dodis and A. Smith. Entropic security and the encryption of high entropy messages. In J. Kilian, editor, TCC 2005, volume 3378 of $L N C S$, pages 556-577. Springer, Heidelberg, Feb. 2005. [

[31] D. M. Freeman, O. Goldreich, E. Kiltz, A. Rosen, and G. Segev. More constructions of lossy and correlation-secure trapdoor functions. In P. Q. Nguyen and D. Pointcheval, editors, PKC 2010, volume 6056 of LNCS, pages 279-295. Springer, Heidelberg, May 2010. 뵤, 미

[32] B. Fuller, A. O'Neill, and L. Reyzin. A unified approach to deterministic encryption: New constructions and a connection to computational entropy. Journal of Cryptology, 28(3):671-717, July 2015.

[33] S. Garg, C. Gentry, S. Halevi, M. Raykova, A. Sahai, and B. Waters. Candidate indistinguishability obfuscation and functional encryption for all circuits. In 54th FOCS, pages 40-49. IEEE Computer Society Press, Oct. 2013. 四, [

[34] C. Gentry, A. B. Lewko, A. Sahai, and B. Waters. Indistinguishability obfuscation from the multilinear subgroup elimination assumption. In V. Guruswami, editor, 56th FOCS, pages 151-170. IEEE Computer Society Press, Oct. 2015. 四

[35] S. Goldwasser and Y. T. Kalai. On the impossibility of obfuscation with auxiliary input. In 46th FOCS,

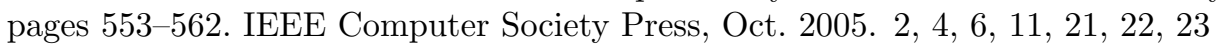

[36] V. Goyal, A. O'Neill, and V. Rao. Correlated-input secure hash functions. In Y. Ishai, editor, TCC 2011, volume 6597 of LNCS, pages 182-200. Springer, Heidelberg, Mar. 2011. [6, [, 미

[37] B. Hemenway and R. Ostrovsky. Building lossy trapdoor functions from lossy encryption. In K. Sako and P. Sarkar, editors, ASIACRYPT 2013, Part II, volume 8270 of LNCS, pages 241-260. Springer, Heidelberg, Dec. 2013. ㅁ, 미

[38] D. Hofheinz, J. Malone-Lee, and M. Stam. Obfuscation for cryptographic purposes. In S. P. Vadhan, editor, TCC 2007, volume 4392 of LNCS, pages 214-232. Springer, Heidelberg, Feb. 2007. ए28

[39] S. Hohenberger, G. N. Rothblum, a. shelat, and V. Vaikuntanathan. Securely obfuscating re-encryption. In S. P. Vadhan, editor, TCC 2007, volume 4392 of LNCS, pages 233-252. Springer, Heidelberg, Feb. 2007. 28

[40] V. Koppula, O. Pandey, Y. Rouselakis, and B. Waters. Deterministic public-key encryption under continual leakage. Cryptology ePrint Archive, Report 2014/780, 2014. http://eprint.iacr.org/ 2014/780.

[41] B. Lynn, M. Prabhakaran, and A. Sahai. Positive results and techniques for obfuscation. In C. Cachin and J. Camenisch, editors, EUROCRYPT 2004, volume 3027 of LNCS, pages 20-39. Springer, Heidel-

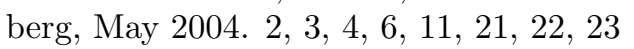

[42] T. Matsuda and G. Hanaoka. Chosen ciphertext security via point obfuscation. In Y. Lindell, editor,

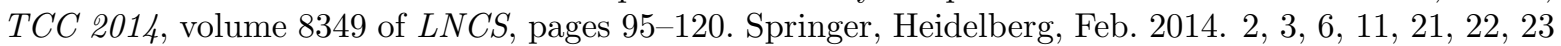

[43] T. Matsuda and G. Hanaoka. Chosen ciphertext security via UCE. In H. Krawczyk, editor, PKC 2014, volume 8383 of $L N C S$, pages 56-76. Springer, Heidelberg, Mar. 2014. 回

[44] I. Mironov, O. Pandey, O. Reingold, and G. Segev. Incremental deterministic public-key encryption. In D. Pointcheval and T. Johansson, editors, EUROCRYPT 2012, volume 7237 of LNCS, pages 628-644. Springer, Heidelberg, Apr. 2012. 


Game $\operatorname{COR}_{\mathrm{Obf}, \mathrm{X}}^{\mathcal{A}}(\lambda)$
$(\mathbf{k}, a) \leftarrow{ }^{\mathrm{X}} \operatorname{\operatorname {Ev}}\left(1^{\lambda}\right)$
$\overline{\mathbf{P}} \leftarrow \$ \operatorname{Obf}\left(1^{\lambda}, \mathbf{I}_{\mathbf{k}}\right)$
$k \leftarrow \AA \mathcal{A}\left(1^{\lambda}, \overline{\mathbf{P}}, a\right)$
$\operatorname{Return}(\exists i: \overline{\mathbf{P}}[i](k)=1)$

Figure 11: Game defining computational correctness of point-function obfuscator Obf.

[45] A. Mittelbach. Salvaging indifferentiability in a multi-stage setting. In P. Q. Nguyen and E. Oswald, editors, EUROCRYPT 2014, volume 8441 of LNCS, pages 603-621. Springer, Heidelberg, May 2014. \&

[46] R. Pass, K. Seth, and S. Telang. Indistinguishability obfuscation from semantically-secure multilinear encodings. In J. A. Garay and R. Gennaro, editors, CRYPTO 2014, Part I, volume 8616 of LNCS, pages 500-517. Springer, Heidelberg, Aug. 2014. 四

[47] C. Peikert and B. Waters. Lossy trapdoor functions and their applications. In R. E. Ladner and C. Dwork, editors, 40th ACM STOC, pages 187-196. ACM Press, May 2008. ㅁ, 미

[48] A. Raghunathan, G. Segev, and S. P. Vadhan. Deterministic public-key encryption for adaptively chosen plaintext distributions. In T. Johansson and P. Q. Nguyen, editors, EUROCRYPT 2013, volume 7881 of $L N C S$, pages 93-110. Springer, Heidelberg, May 2013.

[49] A. Sahai and B. Waters. How to use indistinguishability obfuscation: deniable encryption, and more. In D. B. Shmoys, editor, 46th ACM STOC, pages 475-484. ACM Press, May / June 2014. [

[50] M. Varia. Studies in Program Obfuscation. PhD thesis, Massachusetts Institute of Technology, Cambridge, MA, USA, 2010. [?

[51] H. Wee. On obfuscating point functions. In H. N. Gabow and R. Fagin, editors, 37th ACM STOC,

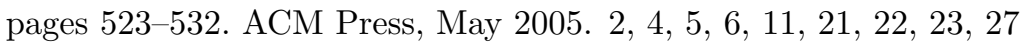

[52] H. Wee. Dual projective hashing and its applications - lossy trapdoor functions and more. In D. Pointcheval and T. Johansson, editors, EUROCRYPT 2012, volume 7237 of LNCS, pages 246-262. Springer, Heidelberg, Apr. 2012. [0, 미

[53] D. Wichs. Barriers in cryptography with weak, correlated and leaky sources. In R. D. Kleinberg, editor, ITCS 2013, pages 111-126. ACM, Jan. 2013. [

[54] X. Xie, R. Xue, and R. Zhang. Deterministic public key encryption and identity-based encryption from lattices in the auxiliary-input setting. In I. Visconti and R. D. Prisco, editors, SCN 12, volume 7485 of LNCS, pages 1-18. Springer, Heidelberg, Sept. 2012. ㅂ, 뚀

[55] H. Xue, B. Li, X. Lu, D. Jia, and Y. Liu. Efficient lossy trapdoor functions based on subgroup membership assumptions. In M. Abdalla, C. Nita-Rotaru, and R. Dahab, editors, CANS 13, volume 8257 of LNCS, pages 235-250. Springer, Heidelberg, Nov. 2013. 15, 묘

\section{A Computational correctness and PO from any UCE}

In Section 2 we required that all obfuscators satisfy perfect correctness condition, meaning that the result of obfuscating any circuit $\mathrm{C}$ must be an equivalent circuit $\overline{\mathrm{C}}$. We now consider a relaxed form of obfusaction correctness with respect to point functions, which we call a computational correctness. It requires that given an obfuscation $\overline{\mathrm{P}}$ of a point function $\mathbf{I}_{k}$, no PT adversary can find $k^{\prime} \neq k$ such that $\overline{\mathrm{P}}\left(k^{\prime}\right)=1$ holds with better than a negligible probability. We will extend the result from Section 5.3 to build a point-function obfuscator satisfiyng computational correctness from a non-injective mUCE. 
COMPUTATIONAL CORRECTNESS OF POINT-FUnCTION OBFUSCATION. Consider game COR of Fig. [1] associated to an obfuscator Obf, a target generator $\mathrm{X}$ and an adversary $\mathcal{A}$. For $\lambda \in \mathbb{N}$ let $\operatorname{Adv}_{\mathrm{Obf}, \mathbf{X}, \mathcal{A}}^{\text {cor }}(\lambda)=\operatorname{Pr}\left[\mathrm{COR}_{\mathrm{Obf}, \mathbf{X}}^{\mathcal{A}}(\lambda)\right]$. Let $\mathbf{X}$ be a class of target generators. We say that Obf is a point-function obfuscation that satisfies computational correctness condition for $\mathbf{X}$ if $\operatorname{Adv}_{\mathrm{Obf}, \mathrm{X}, \mathcal{A}}^{\mathrm{cor}}(\cdot)$ is negligible for every $\mathrm{PT} \mathcal{A}$ and every $\mathrm{X} \in \mathbf{X}$.

Theorem A.1 Let $\mathrm{H}$ be a family of functions such that $2^{\mathrm{H} . \mathrm{il}(\cdot)-\mathrm{H} . \mathrm{ol}(\cdot)}$ is negligible. Let $\mathbf{X}$ be a class of target generators such that $\mathbf{X} . \mathrm{tl}=\mathrm{H}$.il for all $\mathbf{X} \in \mathbf{X}$. Assume $\mathbf{H}$ is $\mathrm{mUCE}\left[\mathbf{S}^{\mathbf{X}}\right]$-secure. Let Obf be the obfuscator as constructed from function family $\mathrm{H}$ in Section 5.3. Then (1) Obf satisfies computational correctness condition for $\mathbf{X}$, and (2) Obf is $\operatorname{IND}[\mathbf{X}]$-secure.

Proof of Theorem [A.1: Proof of part (2) is identical to the proof of security for Theorem 5.3]. We now prove part (1). Let $\mathbf{X} \in \mathbf{X}$ be any target generator with $\mathbf{X}$.tl $=\mathbf{H}$.il. Let $\mathcal{S}^{\mathbf{X}}$ be the corresponding source as defined in Section 5.3. Let $\mathcal{A}$ be a PT adversary against the COR-security of Obf relative to $\mathrm{X}$. We build a PT distinguisher $\mathcal{D}$ as follows:

\begin{tabular}{l|l}
$\frac{\text { Distinguisher } \mathcal{D}\left(1^{\lambda}, \mathbf{h k}, L\right)}{\left(\left(d, a_{1}\right), \mathbf{y}\right) \leftarrow L}$ & $\frac{\text { Circuit } \mathrm{C}_{1^{\lambda}, h k, y}\left(k^{\prime}\right)}{y^{\prime} \leftarrow \mathrm{H} . \operatorname{Ev}\left(1^{\lambda}, h k, k^{\prime}\right)}$ \\
For $i=1, \ldots,|\mathbf{y}|$ do $\overline{\mathbf{P}}[i] \leftarrow \mathrm{C}_{1^{\lambda}, \mathbf{h k}[i], \mathbf{y}[i]}$ & If $\left(y=y^{\prime}\right)$ then return 1 \\
$k \leftarrow \$ \mathcal{A}\left(1^{\lambda}, \overline{\mathbf{P}}, a_{1}\right)$ & Else return 0 \\
If $(\exists i: \overline{\mathbf{P}}[i](k)=1)$ then return 1 & \\
Else return 0 &
\end{tabular}

Let $\lambda \in \mathbb{N}$. Let $b$ denote the challenge bit in game $\operatorname{mUCE}_{\mathrm{H}}^{\mathcal{S}^{\times}, \mathcal{D}}(\lambda)$, and let $b^{\prime}$ denote the bit returned by $\mathcal{D}$ in the same game. We claim that

$$
\operatorname{Pr}\left[b^{\prime}=1 \mid b=1\right] \geq \frac{1}{2} \cdot \operatorname{Adv}_{\mathrm{Obf}, \mathrm{X}, \mathcal{A}}^{\mathrm{cor}}(\lambda) \quad \text { and } \operatorname{Pr}\left[b^{\prime}=1 \mid b=0\right] \leq \mathrm{X} \cdot \mathrm{vl}(\lambda) \cdot \frac{2^{\mathrm{H} \cdot \mathrm{il}(\lambda)}}{2^{\mathrm{H} . \mathrm{ol}(\lambda)}} .
$$

Consider the "real" case of mUCE game, meaning $b=1$. Note that source $\mathcal{S}^{\mathrm{X}}$ uses targets generated by $\mathrm{X}$ with probability $\frac{1}{2}$. Whenever this happens, adversary $\mathcal{D}$ can use $\mathcal{A}$ to recover a valid target for some of the point-function obfuscations with probability $\operatorname{Pr}\left[\mathrm{COR}_{\text {Obf, } \mathrm{X}}^{\mathcal{A}}(\lambda)\right]$. $\mathcal{D}$ returns 1 iff $\mathcal{A}$ was succesful. This gives us the first equation.

Next, consider the "random" case of mUCE game, meaning $b=0$. Let $i \in\{1, \ldots, \mathrm{X} . \mathrm{vl}(\lambda)\}$. Note that the value of $\mathbf{y}[i]$ is chosen uniformly at random from $\{0,1\}^{\mathrm{H} . \mathrm{ol}(\lambda)}$ and is independent of $\mathbf{h k}[i]$. Therefore, $\mathbf{y}[i]$ belongs to the range of $\mathbf{H}\left(1^{\lambda}, \mathbf{h k}[i], \cdot\right)$ with probability $2^{\mathrm{H} . \mathrm{il}(\lambda)-\mathrm{H} . \mathrm{ol}(\lambda)}$. If $\mathbf{y}[i]$ is outside the range, then $\mathcal{A}$ can not produce a valid $k$ and hence $\mathcal{D}$ returns 0 . We get the second equation by applying the union bound for all possible values of $i \in\{1, \ldots, \mathrm{X} \cdot \mathrm{vl}(\lambda)\}$.

Combining the two equations together, we have

$$
\operatorname{Adv}_{\mathrm{Obf}, \mathrm{X}, \mathcal{A}}^{\mathrm{cor}}(\lambda) \leq 2 \cdot \operatorname{Adv}_{\mathrm{H}, \mathcal{S}^{\times}, \mathcal{D}}^{\mathrm{m}-\mathrm{uce}}(\lambda)+\mathrm{X} \cdot \mathrm{vl}(\lambda) \cdot 2^{\mathrm{H} . \mathrm{il}(\lambda)-\mathrm{H} . \mathrm{ol}(\lambda)+1},
$$

for all $\lambda \in \mathbb{N}$, where $\operatorname{Adv}_{\mathrm{H}, \mathcal{S}^{\times}, \mathcal{D}}^{\mathrm{m}-\mathrm{uce}}(\cdot)$ and $2^{\mathrm{H} . \mathrm{il}(\cdot)-\mathrm{H} . \mathrm{ol}(\cdot)}$ are assumed to be negligible. Therefore, obfuscator Obf satisfies computational correctness condition for $\mathbf{X}$. 